\title{
Moral Hazard and Monopolistically Competitive Insurance Markets
}

\author{
by Martin F. Hellwig *
}

\section{Moral hazard and quantity rationing in insurance contracts}

Insurance reduces the incentive to take care against accidents. This phenomenon is known as moral hazard. In his lecture, Professor Stiglitz presents a thorough analysis of the implications of moral hazard for competitive insurance markets. This analysis parallels the earlier work of Rothschild and Stiglitz [1976] and of Wilson [1977] on adverse selection and competitive insurance markets.

Under moral hazard, the total amount of insurance purchased by an agent determines the effort he takes to prevent an accident and therefore the probability of an accident. The expected profit on the sale of one unit of insurance will therefore depend on the total amount of insurance purchased by the agent so that insurance is not a homogeneous good. A similar situation occurs in credit markets where the return on a loan depends on the probability of bankruptcy, which in turn depends on the borrower's total indebtedness.

From a welfare point of view, moral hazard will typically make perfect insurance undesirable because an agent who is perfectly insured does not care whether an accident takes place and therefore does nothing to prevent it. A "second best" insurance system should provide partial insurance up to the point where the benefits of additional risk sharing are outweighed by the cost arising from reduced incentives for accident prevention (Pauly [1974], Shavell [1979]).

From a positive point of view, the behaviour of a competitive insurance market depends crucially on the feasibility of imposing a nonlinear premium-benefit schedule or, equivalently, of rationing the total quantity of insurance available to an individual. If such quantity rationing is feasible, a competitive insurance market with free entry

* Professor of economic theory, University of Bonn. 
of firms will offer the "second best" feasible insurance contract, which provides the optimal amount of insurance at fair odds (Helpman and Laffont [1975], Shavell [1979]).1

If the total quantity of insurance available to an individual cannot be rationed, a competitive market with free entry of firms will never provide the "second best" insurance contract (Pauly [1974]). Now Professor Stiglitz argues that such a market may not have an equilibrium at all, or that it may have an equilibrium with positive profits. This analysis is based on the observation that consumer behaviour may be discontinuous, e.g. because a small reduction in the premium-benefit ratio leads to an abrupt substitution of insurance for accident prevention. ${ }^{2}$

I have two difficulties with these propositions. Both relate to Professor Stiglitz's treatment of the feasibility of quantity rationing as an exogenous characteristic of the insurance market. First, the feasibility of quantity rationing depends largely on the information firms have about their customers' contracts. Quantity rationing is certainly feasible if all firms inform each other about all contracts that they conclude. As Jaynes [1978] pointed out in the context of adverse selection, the absence or presence of such communication among firms should not be postulated exogenously. Instead, we must ask what profit incentives firms have to share or conceal information about their customers.

Second, quantity rationing may be feasible even if firms fail to share information about their customers. In any event, each company can control the amount of insurance it sells to its own customers. If it turned out that in equilibrium only a few firms are active in the insurance market, one would obtain a form of quantity rationing simply because consumers have only few firms from which they can buy insurance.

To see the importance of this argument, consider Professor Stiglitz's proposition that the insurance market may have no equilibrium at all. Suppose for instance that the minimum effort level corresponds to the case where the consumer sets the fire himself without being found out, and the accident occurs with probability one. If there is no quantity rationing, and the consumer can buy an arbitrary amount of insurance at a finite premium-benefit ratio, he will find it optimal to buy an infinite amount of insurance and then set his house afire so as to collect his benefits with probability one. This situation cannot be an equilibrium because the insurance companies lose money. In contrast, if no insurance is available, a single firm which does control the amount of insurance it sells to its own customers will find it profitable to enter the market with

I Strictly speaking, this statement is correct only in a partial equilibrium analysis of the insurance market. I neglect the general equilibrium interactions between markets that Professor Stliglitz discusses in Section 4 of his paper. Since moral hazard precludes the attainment of a first best allocation, an appropriate system of specific taxes and subsidies may improve the allocation by taking account of externalities between different markets. However, it is unclear what taxes would actually be required.

2 This observation is due to Helpman and Laffont [1975] who found that equilibrium need not exist if consumers can buy negative as well as positive insurance and, moreover, the insurance companies are restricted to announcing premium-benefit ratios at which consumers can buy arbitrary amounts of insurance. This nonexistence result disappears, if insurance purchases must be nonnegative. The equilibrium premium-benefit ratio then is simply that which maximizes the consumer's expected utility subject to the constraint that profits be nonnegative. 
a contract that is "small" enough to preserve an incentive for accident prevention. Therefore, zero insurance cannot be an equilibrium either.

However, at this point, the conclusion that the market has no equilibrium is premature. The preceding arguments rule out equilibria in which the consumer can buy arbitrary amounts of insurance as well as equilibria in which the consumer can buy no insurance at all. They simply do not cover the possibility that the consumer can buy a limited amount of insurance from a finite number of insurance companies.

In the following, I shall rconsider Professor Stiglitz's propositions in the light of these criticisms. The main conclusions of the analysis can briefly be summarized as follows :

- Typically, the insurance market has no equilibrium in which all firms find it profitable to share information about their customers.

- If effort has only two possible values, there always exists an equilibrium in which active communication among firms with the most favourable premium-benefit ratio makes quantity rationing feasible.

- Equilibrium profits may be positive even in equilibria with active communication among firms.

- If efforts has only two possible values and consumers have constant absolute risk aversion, there may exist equilibria without communication in which quantity rationing is effective because a finite number of insurance companies offer only a limited amount of insurance. In general, the question of nonexistence of equilibrium remains open.

\section{Monopolistically competitive equilibria with endogenous quantity information}

I shall begin with an informal discussion of the profit incentives for communication among firms. The first firm to conclude an insurance contract with a given agent will always make this information available to other firms. Other firms then know that the agent has less incentive to take care and has a higher accident probability than an agent without insurance. This information reduces their incentive to offer insurance to the agent, which is precisely what the first firm wants to achieve.

The situation of a second firm offering supplementary insurance is less clear. On the one hand, this firm has the same incentive as the first firm to publicize information about its contracts in order to discourage further supplementary insurance contracts. On the other hand, the first insurance contract may become invalid if the existence of a supplementary insurance contract becomes known. In this case the agent will not accept supplementary insurance unless it is kept secret. ${ }^{3}$ The behaviour of firms selling supplementary insurance will depend on the presumed attitudes of firms with "prior" contracts to such supplementary insurance.

3 Ideally then, the second insurance company would like to divulge information about its contracts to all but the first insurance company. For simplicity, I assume that such partial sharing of information is infeasible. This assumption has no effect on the results below. 
To analyse this question in detail, one must specify strategies and payoffs for all firms. A contract offer by a firm to a customer will be a pair $(\gamma, s)$, where $\gamma=(\alpha, \beta) \in \mathbb{R}_{+}^{2}$ indicates the net insurance benefit $\alpha$ and the premium $\beta$, and $s \in\{0,1\}$ indicates whether the firm promises to keep the contract secret or not. A firm's contract offer to an agent will depend on whatever other contracts the agent is known to have concluded. Without loss of generality, one may assume that the firm cares only about the aggregate net benefit and premium on those other contracts. The firm's strategy will then be a function $g: \mathbb{R}_{+}^{2} \rightarrow \mathbb{R}_{+}^{2} \times\{0,1\}$, which shows how the firm's contract offer depends on its information about the agent's other insurance. ${ }^{4}$

To model freedom of entry, I shall allow for countable number of insurance companies, $i=1,2, \ldots$. Given an array of firm strategies, $G=\left(g_{1}, g_{2}, \ldots\right)$, a consumer must choose what contract offers to accept. A sequence $\bar{c}=\left\{\bar{\gamma}_{i}\right\}_{i=1}^{\infty} \in\left(\mathbb{R}_{\dagger}^{2}\right)^{\infty}$ of contract combinations is said to be feasible, if and only if there exists subsets $I_{0}, I_{1}$ of firms, such that :

$$
\begin{aligned}
& \mathrm{i}: \text { for } i \in I_{0},\left(\bar{\gamma}_{i}, 0\right)=g_{i}\left(\sum_{j \in J_{1}} \bar{\gamma}_{j}\right), \\
& \text { ii : for } i \in I_{1},\left(\bar{\gamma}_{i}, 1\right)=g_{i}\left(\sum_{j \in I_{1}>\{i\}} \bar{\gamma}_{j}\right), \\
& \text { iii : for } i \notin I_{0} \cup I_{1}, \bar{\gamma}_{i}=0 .
\end{aligned}
$$

The set of feasible contract combinations is denoted as $\mathrm{C}(G)$. Given $G$, the consumer chooses an effort level $e$ from a feasible set $E$ and a feasible contract combination $\bar{c}$ to solve the problem : ${ }^{5}$

$$
\begin{aligned}
& \operatorname{Max}_{e \in E}(1-p(e)) u\left(w-\sum_{i} \bar{\beta}_{i}\right)+p(e) u\left(w-d+\sum_{i} \bar{\alpha}_{i}\right)-e \\
& \bar{c}=\left\{\bar{\alpha}_{i}, \bar{\beta}_{i}\right\} \in \mathrm{C}(G)
\end{aligned}
$$

Given the consumer's choice $(e, \bar{c})$, firms' expected profits are given by the sequence $\bar{\pi}(e, \bar{c})=\left\{\pi_{i}(e, \bar{c})\right\}_{i=1}^{\infty}$, where

$$
\bar{\pi}_{i}(e, \bar{c})=(1-p(e)) \bar{\beta}_{i}-p(e) \bar{\alpha}_{i} \text {. }
$$

Let $\sigma(G)$ be the set of maximizers for problem (1), and let $\psi(G)$ be the set of profit sequences $\bar{\pi}(e, \bar{c})$ that correspond to some $(e, \bar{c}) \in \emptyset(G)$. This set $\psi(G)$ contains the payoff allocations corresponding to the strategy array $G$.

Equilibrium can now be defined in the usual fashion. Given an array of strategies $G=\left(g_{1}, g_{2}, \ldots\right)$ and another strategy $h$, let $H_{i}(h, G)$ be the new array of strategies that arises if firm $i$ chooses strategy $h$ whereas firms $j \pm i$ continue to use the strategies $g_{j}$. A monopolistically competitive equilibrium (MCE) for the insurance market will be an array of strategies $G$ and a profit sequence $\bar{\pi}$ with the following properties:

i : $\bar{\pi} \in \psi(G)$;

ii : $\bar{\pi}_{i} \geqslant 0$ for all $i$;

iii : for all $i$ and all strategies $h \pm g_{i}, \hat{\pi} \in \psi\left(H_{i}(h, G)\right)$ implies $\hat{\pi}_{i} \leqslant \bar{\pi}_{i}$.

${ }^{4}$ More generally, the firm might let the consumer choose among a set of contracts (see e.g. Jaynes [1978]). Restricting firm strategies to functions rather than correspondences simplifies the analysis without affecting the results. The last point is obvious once one observes that any set of contract offers by a single firm can also be obtained as a vector of contract offers by different firms.

${ }^{5}$ The interpretation of problem (1) is the same as in the lecture by Professor Stiglitz. 
An MCE is said to involve potential entry, if at least one firm $i$ is completely inactive and has the constant strategy $g_{i}(\gamma)=(0,0,0)$ for all $\gamma \in \mathbb{R}_{+}^{2}$.

Except for the explicit treatment of the transmission and the use of information, the concept of an MCE with potential entry is the same as the equilibrium concept used by Professor Stiglitz for the case when insurance purchases are not observable. ${ }^{6}$ In fact, every equilibrium in his analysis can be represented as an MCE in which firms do not communicate with each other: If $\gamma_{i}^{*}, \pi_{i}^{*}$ are the equilibrium insurance terms and the equilibrium expected profits of firm $i$, let $g_{i}^{*}$ be the strategy with the constant value $\left(\gamma_{i}^{*}, 0\right)$, and consider the array $G^{*}=\left(g_{1}^{*}, g_{2}^{*}, \ldots\right)$. Clearly, $\left\{\pi_{i}^{*}\right\} \in \psi\left(G^{*}\right)$, and $\pi_{i}^{*} \geqslant 0$ for all $i$. Moreover, since the other firms do not react to any information, no firm can improve its position by sharing information about its contracts. Given that the other firms do not share any information about their customers, no firm can improve its position by using a non constant rather than a constant strategy. Finally, by assumption, $g_{i}^{*}$ with the constant value $\left(\gamma_{i}^{*}, 0\right)$ is firm $i$ 's best constant value strategy given that the other firms use the strategies $g_{j}^{*}, j \pm i$. The pair $G^{*},\left\{\pi_{i}^{*}\right\}$ must therefore be an MCE. ${ }^{7}$

There is something implausible about the preceding argument. No firm shares information because no firm uses information; no firm uses information because no firm shares information. This sort of argument will also prove that one cannot have a telephone system because nobody is willing to acquire the first telephone. The first person to acquire a telephone has nobody to talk to, so that the acquisition is not worth while.

As in the case of the telephone system, one suspects that the equilibria without communication are not robust once firms begin to experiment to see what happens if they do communicate after all. If some firm begins to share information about its customers, one would expect other firms to begin using nonconstant strategies that exploit the information they receive.

In the following section, I will make this notion of non-robustness of an equilibrium without communication precise in order to determine what communication patterns and what insurance allocations will eventually emerge.

6 Professor Stiglitz is not explicit about firms' strategies. However, his statement that "if no insurance is offered, it will pay some firm to offer a small policy" (p. 21) suggests that each firm $i$ determines the amount of insurance, i.e. the pair $\left(\alpha_{i}, \beta_{i}\right)$, that it offers to a customer (see also fn 23 , p. 20). To accommodate the notion that individuals can buy arbitrary amounts of insurance " at market terms" (p. 22), i.e. arbitrary multiples of a given unit contract $\gamma_{0}$, one might have a sequence of firms $\left\{i_{k}\right\}_{k=1}^{\infty}$, such that firm $i_{k}$ offers the contract $\frac{1}{k} \gamma_{0}$, and the consumer can combine his contracts so as to obtain any point on the ray $\left\{\lambda \gamma_{0} \mid \lambda \geqslant 0\right\}$. To be sure, this sequence would involve what Stiglitz calls " latent" contract offers. However, without "latent" contract offers, I do not understand what it means to "supplement this by buying at market terms ", nor do I understand his argument for the existence of equilibria with positive profits.

${ }^{7}$ Note that the argument breaks down if firms communicate and choose strategies with the constant values $\left(\gamma_{i}^{*}, 1\right)$ rather than $\left(\gamma_{i}^{*}, 0\right)$. In this situation, an entering firm can make positive profits by offering insurance terms close to the "second best" amount of insurance on condition that no additional insurance is purchased. Since all other firms share information about their customers, the entering firm can be sure to have only customers with no additional insurance. Moreover, consumers will accept the entering firm's offer because they prefer the "second best" contract to what they could otherwise get in the market. I shall return to this point in section 4 below. 


\section{Positive profits and the incentive to share information}

It will be useful to consider Professor Stiglitz's positive profit equilibria. If we represent these equilibria as MCE's without communication, they have the following structure. There is an infinite sequence of firms $\left\{i_{k}\right\}_{k=1}^{\infty}$, such that firm $i_{k}$ has the strategy $g_{i_{k}}^{*}$ with the constant value $\left(\frac{1}{k} \gamma_{0}^{*}, 0\right)$ where $\gamma_{0}^{*}=\left(\alpha_{0}^{*}, \beta_{0}^{*}\right)$ is fixed. No firm offers contracts with a premium-benefit ratio less than $\beta_{0}^{*} / \alpha_{0}^{*}$. The consumer buys the contract $\left(\gamma_{0}^{*}, 0\right)$ from firm $i_{1}$ and takes a lot of effort, so that firm $i_{1}$ makes positive profits. However, the consumer would also be willing to combine the contracts $\left(\frac{1}{k} \gamma_{0}^{*}, 0\right), k=1,2, \ldots$, so as to to be overinsured and then take no effort at all.

In this situation, no firm $j$ tries to displace firm $i_{1}$ with a contract offer $\left(\gamma_{j}, s_{j}\right)$ involving a premium-benefit ratio less than $\beta_{0}^{*} / \alpha_{0}^{*}$. If any such contract was available, the consumer would accept it and then strictly prefer to supplement it from the contracts $\left(\frac{1}{k} \gamma_{0}^{*}, 0\right)$ in order to overinsure and then take no effort at all. Aggregate profits would then be negative. The contract $\left(\gamma_{j}, s_{j}\right)$, which has the lowest premium-benefit ratio, would certainly make a loss.

Suppose that the entering firm $j$ sets $s_{j}=1$ and publicizes its customers. In this case, the preceding argument depends crucially on the constancy of the strategy functions $g_{i k}^{*}$. The contracts $\left(\frac{1}{k} \gamma_{0}^{*}, 0\right)$ must remain available to the consumer even if he is known to have purchased the contract $\left(\gamma_{j}, s_{j}\right)$.

This behaviour by the firms $i_{k}, k=1,2, \ldots$, is implausible. For suppose that the entering firm chooses $\gamma_{j}$ close to $\gamma_{0}^{*}$, so it has almost the same premium-benefit ratio as the other firms. Then the firms $i_{k}, k=1,2, \ldots$, will bear at least some of the losses that arise when the consumer overinsures and takes no effort. Under the constant strategies $g_{i_{k}}^{*}$, the firms $i_{k}, k=1,2, \ldots$, offer the contracts $\left(\frac{1}{k} \gamma_{0}^{*}, 0\right)$ to a consumer who is known to have purchased the contract $\left(\gamma_{j}, 1\right)$, even though they know that he will choose a contract combination at which they will lose money. To choose these strategies, they must be confident that the contract $\left(\gamma_{j}, 1\right)$ will never be available to the consumer.

The availability of the contracts $\left(\frac{1}{k} \gamma_{0}^{*}, 0\right)$ may be regarded as a threat against entering firms. The firms $i_{k}, k=1,2, \ldots$, announce this threat even though they would not like to carry it out if entry did occur. They hope that the threat will successfully discourage entry and will not have to be carried out. Professor Stiglitz's positive profit equilibria thus depend on a bluff that is not called. If by some chance the bluff were called and entry did occur, such an equilibrium would probably fall apart because the firm $i_{k}$ would withdraw their contract offers after all. The threat against entry that is implicit in the availability of these contracts is not credible.

To avoid this peculiar vulnerability of Professor Stiglitz's positive profit equilibria, I shall impose an additional condition of perfectness ${ }^{8}$ on the equilibrium array of strategies

${ }^{8}$ The perfect equilibrium concept is originally due to Selten. The state of the current game-theoretic discussion of this concept may be gathered from Kreps and Wilson [1982]. 
$G=\left(g_{1}, g_{2}, \ldots\right)$. If by some chance it becomes known that the consumer has an additional insurance contract $\left(\gamma_{0}, 1\right)$, the firms $1,2, \ldots$ should not find it desirable to abandon their strategies $g_{1}, g_{2}, \ldots$ and withdraw from the market altogether.

Formally, a sequence $\bar{c}=\left\{\bar{\gamma}_{i}\right\}_{i=1}^{\infty}$ of contract combinations is called a feasible supplement to the contract $\left(\gamma_{0}, 1\right)$, if and only if there exist subsets $I_{0}, I_{1}$ of firms, such that :

$$
\begin{aligned}
& \mathrm{i}: \text { for } i \in I_{0},\left(\bar{\gamma}_{i}, 0\right)=g_{i}\left(\gamma_{0}+\sum_{j \in L_{1}} \bar{\gamma}_{j}\right), \\
& \text { ii : for } i \in I_{1},\left(\bar{\gamma}_{i}, 1\right)=g_{i}\left(\gamma_{0}+\sum_{j \in I_{i}>\{i\}} \bar{\gamma}_{j}\right), \\
& \text { iii : for } i \notin I_{0} \cup I_{1}, \bar{\gamma}_{i}=0 .
\end{aligned}
$$

The set of feasible supplements to the contract $\left(\gamma_{0}, \mathrm{l}\right)$ is denoted as $\mathrm{C}\left(\gamma_{0}, G\right)$. Given $\gamma_{0}=\left(\alpha_{0}, \beta_{0}\right)$ and $G$, the consumer chooses an effort level $e$ and a feasible supplement $\bar{c}$ to the contract $\left(\gamma_{0}, 1\right)$ to solve the problem :

$$
\begin{aligned}
& \operatorname{Max}_{\ell \in E}(1-p(e)) u\left(w-\beta_{0}-\sum_{i} \bar{\beta}_{i}\right)+p(e) u\left(w-d+\alpha_{0}+\sum_{i} \bar{\alpha}_{i}\right)-c \\
& \bar{c}=\left\{\bar{\alpha}_{i}, \bar{\beta}_{i}\right\} \in \mathrm{C}\left(\gamma_{0}, G\right)
\end{aligned}
$$

Given the consumer's choice $(e, \bar{c})$, firm $i$ 's expected profit is again $\bar{\pi}_{i}(e, \bar{c})=(1-p(e)) \bar{\beta}_{i}-p(e) \bar{\alpha}_{i}$, as in (2). Let $\vartheta\left(\gamma_{0}, G\right)$ be the set of maximizers for problem (3), and let $F(G)$ be the set of $\gamma_{0} \in \mathbb{R}_{+}^{2}$ for which the solution to problem (3) is strictly better than the solution to problem (1). An MCE $G, \bar{\pi}$ will be called perfect if for all $\gamma_{0} \in F(G)$ there exists $(e, \bar{c}) \in \emptyset\left(\gamma_{0}, G\right)$ such that $\bar{\pi}_{i}(e, \bar{c}) \geqslant 0$ for all $i$.

For perfect MCE's, I shall exhibit the conscious use of communication as a tool of competition. A firm that discloses its contracts to other firms will reduce the supplementary insurance available to its customers. The customer will therefore take more care to prevent accidents, and the firm can compete by lowering its premium-benefit ratio.

For $(v, q) \in \mathbb{R}_{+}^{2}$, let $\lambda(v, q)$ be the set of triples $(e, \alpha, \beta)$ that maximize the objective $(1-p(e)) u(w-\beta)+p(e) u(w-d+\alpha)-e$ under the constraints $\beta=q \alpha-v$ and $0 \leqslant \beta \leqslant w$. An MCE is called a price equilibrium, if the equilibrium effort level and aggregate insurance $\left(e, \sum \bar{\gamma}_{i}\right)$ belong to $\lambda(0, \bar{q})$ for $q=\sum \bar{\beta}_{i} / \sum_{i} \bar{\alpha}_{i}$, the equilibrium aggregate premium-benefit ratio. All equilibria that were discussed by Professor Stiglitz are price equilibria.

Let $\underline{e}$ be the minimum feasible effort level, $\bar{p}=p(\underline{e})$ the maximum accident probability, and $\bar{q}=\bar{p} /(1-\bar{p})$ the break-even premium-benefit ratio corresponding to the accident probability $\bar{p}$. The discussion in the beginning of this section is now summarized in the following proposition whose formal proof is given in the appendix.

Proposition 1: A price equilibrium with potential entry is perfect, if and only if the equilibrium aggregate premium-benefit ratio is equal to $\bar{q}$.

Except for the limiting case when the demand correspondence $\lambda$ is discontinous at the price $\bar{q}$ itself, Proposition 1 implies that Professor Stiglitz's positive equilibria are not perfect. These equilibria disappear as soon as the potential entrants realize that the other firms' announced strategies entail losses if entry does occur.

However, a modification of Professor Stiglitz's argument yields another class of MCE's with potential entry and positive profits that are perfect, but that are not price equilibria. Like Professor Stiglitz, I shall exploit the discontinuity of the correspond- 
ence $\lambda$. However, whereas he works with the price-demand correspondence $\lambda(0,),. \mathrm{I}$ shall instead work with the income-demand correspondence $\lambda(., \bar{q})$ at the premiumbenefit ratio $\bar{q}$.

Proposition 2: Assume that for all $v \geqslant 0, \quad(e, \alpha, \beta) \in \lambda(v, \bar{q})$ implies $(1-p(e)) \beta-p(e) \alpha \pm 0$. Then there exists a perfect MCE with potential entry, $(G, \bar{\pi})$, such that $\sum_{i} \bar{\pi}_{i}>0$.

Proposition 2 is based on the following lemma, which is proved in the appendix. The lemma asserts that the income-demand correspondence $\lambda(., \vec{q})$ at the price $\vec{q}$ has essentially the shape shown in Figure 1. In particular, there exists an income level $v_{0}$ at which $\lambda(., \bar{q})$ jumps into the region of negative profits without intersecting the zero profit line $0,0^{\prime} .{ }^{9}$

Lemma 2.1: Assume that for all $v \geqslant 0,(e, \alpha, \beta) \in \lambda(v, \bar{q})$ implies $(1-p(e)) \beta-p(e) \alpha \pm 0$. Then there exist $v_{0}>0$ and $\left(e_{0}, \alpha_{0}, \beta_{0}\right) \varepsilon \lambda\left(v_{0}, \bar{q}\right)$, such that :

(i) $\left(1-p\left(e_{0}\right)\right) \beta_{0}-p\left(e_{0}\right) \alpha_{0}>0, \quad$ and (ii) for $v>v_{0}, \quad(e, \alpha, \beta) \in \lambda(v, \bar{q}), \quad$ if and only if $\quad e=\underline{e}, \beta=\max \left(0, \frac{\bar{q} \delta-v}{1+\bar{q}}\right), \quad \alpha=\frac{v}{\bar{q}}+\min \left(0, \frac{d \bar{q}-v}{\bar{q}(1+\bar{q})}\right), \quad$ and $\quad$ hence $(1-p(e)) \beta-p(e) \alpha<0$.

In the equilibria of Proposition 2, there will be three sets of firms. Firms $1,4,7, \ldots$ all offer the insurance terms $\left(\alpha_{0}, \beta_{0}\right)$ corresponding to the discontinuity of $\lambda(., \bar{q})$ in Lemma 2.1 and Figure 1. Moreover, they disclose their contracts, and they deny insurance to anybody who is known to have purchased insurance elsewhere. Their equilibrium strategies are :

$$
\begin{aligned}
& g_{i}(\gamma)= \begin{cases}\left(\left(\alpha_{0}, \beta_{0}\right), 1\right) & \text { if } \gamma=(0,0) \\
((0,0,), 0) & \text { if } \gamma \pm(0,0),\end{cases} \\
& i=3 k+1, \quad k=0,1,2, \ldots .
\end{aligned}
$$

Firms 2, 5, 8, .. make unconditional contract offers at the common premium-benefit ratio $\bar{q}$. In particular, firm $i=3 k-1$ offers the contract $\left(\frac{1}{k}(1, \bar{q}), 0\right)$, promising to keep their customers' identities secret. Their equilibrium strategies are :

$$
\begin{array}{ll}
g_{i}(\gamma)=\left(\frac{1}{k}(1, \bar{q}), 0\right) & \text { for all } \gamma \in \mathbb{R}_{+}^{2}, \\
i=3 k-1, \quad k=1,2, \ldots . &
\end{array}
$$

Finally, firms $3,6,9, \ldots$ are the potential entrants and do not offer anything, i.e. :

$$
\begin{aligned}
& g_{i}(\gamma)=((0,0), 0) \quad \text { for all } \gamma \in \mathbb{R}_{+}^{2} \\
& i=3 k, \quad k=1,2, \ldots .
\end{aligned}
$$

Given these strategies, the consumer can buy at most one of the contracts $\left(\left(\alpha_{0}, \beta_{0}\right), 1\right)$ offered by firms $1,4,7, \ldots$. Once he buys such a contract, his purchase is revealed to all firms, and he becomes ineligible for the other contracts $\left(\left(\alpha_{0}, \beta_{0}\right), 1\right) .{ }^{10}$ However, he can

${ }^{9}$ One can use a revealed preference argument to show that the constellation of Figure 1 implies that of Figure 10a in Professor Stiglitz's lecture. The converse need not be true.

10 This is the point where communication makes the difference in comparison to the analysis of Professor Stiglitz. 
secretly buy supplementary insurance at the premium-benefit ratio $\bar{q}>\beta_{0} / \alpha_{0}$. The total amount of such supplementary insurance that he buys is arbitrary.

The aggregate premium-benefit combinations available to the consumer are thus described by the set $\left\{(\alpha, \beta) \mid(\alpha, \beta)=\left(\alpha_{0}, \beta_{0}\right)+\delta(1, \bar{q}), \delta \geqslant 0\right\}$. In Figure 1 , this set corresponds to the straight line with the slope $\bar{q}$ that begins at the point $\left(\alpha_{0}, \beta_{0}\right)$. By Lemma 2.1 we know that on this straight line, the consumer (weakly) prefers the point $\left(\alpha_{0}, \beta_{0}\right)$ itself in combination with the effort level $e_{0}$. We may therefore assume that he chooses the effort level $e_{0}$ and the contract combination $\bar{c}$ with $\bar{\gamma}_{1}=\left(\alpha_{0}, \beta_{0}\right), \bar{\gamma}_{i}=(0,0)$ for $i \pm 1$. This choice yields the expected profits $\bar{\pi}_{1}=\left(1-p\left(e_{0}\right)\right) \beta_{0}-p\left(e_{0}\right) \alpha_{0}>0$, by Lemma 2.1 , and $\bar{\pi}_{i}=0$ for $i \pm 1$.

It remains to show that no firm can gain by changing its strategy. Any firm that wishes to improve its position must try to attract the consumer with a premium-benefit ratio less than $\bar{q}$ that allows him to reach a point like $\left(\alpha_{1}, \beta_{1}\right)$, which is preferred to $\left(\alpha_{0}, \beta_{0}\right)$. ${ }^{11}$ From the point $\left(\alpha_{1}, \beta_{1}\right)$, the consumer can still buy arbitrary amounts of supplementary insurance at the constant premium-benefit ratio $\bar{q}$. The aggregate premium-benefit combinations available to him are now given by the set $\left\{(\alpha, \beta) \mid(\alpha, \beta)=\left(\alpha_{1}, \beta_{1}\right)+\delta(1, \bar{q}), \delta \geqslant 0\right\}$, which corresponds to the straight line with the slope $\bar{q}$, that begins at $\left(\alpha_{1}, \beta_{1}\right)$. By Lemma 2.1, the consumer will now choose the point $\left(\alpha_{2}, \beta_{2}\right)$ and the effort level $e_{2}=e$. ${ }^{12}$ At this point, all contracts with a premium-benefit ratio less than $\vec{q}$ make a loss, whereas the supplementary contracts with the premium-benefit ratio $\bar{q}$ break even. The firm that brings the consumer to the point $\left(\alpha_{1}, \beta_{1}\right)$ has a premium-benefit ratio less than $\bar{q}$, and makes a loss. Hence no firm can gain by deviating from its equilibrium strategy.

Perfectness of the equilibrium follows trivially from the fact that supplementary insurance at the premium-benefit ratio $\bar{q}$ will always at least break even because the accident probability does not exceed $\bar{p}$. Note that this premium-benefit ratio on the contract $\left(\left(\alpha_{0}, \beta_{0}\right), 1\right)$. This is possible only because the communication among firms prevents the consumer from more than one contract $\left(\left(\alpha_{0}, \beta_{0}\right), 1\right){ }^{13}$

\section{Communication, secrecy, and the existence of equilibrium}

I now turn to the proposition that a competitive insurance market may not have an equilibrium at all. Here again, voluntary communication will be shown to have a large effect on the insurance market. Even so, we should not expect all firms to share information about their customers. In fact, I shall argue that some firms will always have an incentive to conceal the contracts they conclude.

1 This can be achieved either by the outright offer $\left(\left(\alpha_{1}, \beta_{1}\right), 1\right)$ or by the supplementary offer $\left(\left(\alpha_{1}-\alpha_{0}, \beta_{1}-\beta_{0}\right), 0\right)$.

${ }^{12}$ Since $\left(\alpha_{i}, \beta_{1}\right)$ and the associated effort level are strictly preferred to $\left(e_{0}, \alpha_{0}, \beta_{0}\right)$, one has $v_{1}=\bar{q} \alpha_{1}-\beta_{1}>v_{0}$.

${ }^{13}$ In contrast, supplementary insurance in Professor Stiglitz's positive profit equilibria has the same premium-benefit ratio as the actual insurance purchase, $\sum_{i} \bar{\beta}_{i} / \sum_{i} \bar{\alpha}_{i}$. 
Let $\left(e^{*}, \alpha^{*}, \beta^{*}\right)$ be the " second best" insurance allocation, i.e. the solution to the problem $\max _{e, \alpha, \beta}(1-p(e)) u(w-\beta)+p(e) u(w-d+\alpha)-e$ subject to $(1-p(e)) \beta-p(e) \alpha \geqslant 0$. In Figure 2 , the pair $\left(\alpha^{*}, \beta^{*}\right)$ is shown as a point of tangency between the zero-profit line $00^{\prime}$ and the consumer's indifference curve. Assume that $\left(e^{*}, \alpha^{*}, \beta^{*}\right)$ involves less than full insurance, i.e. $e^{*}>e$ and $w-\beta^{*}>w-d+\alpha^{*}$, and that the function $p(\cdot)$ is strictly convex. At the point $\left(\alpha^{*}, \overline{\beta^{*}}\right)$ then, a further increase in the net benefit $\alpha$ or in the premium $\beta$ will strictly lower the desired level $e$ and raise the accident probability $p(e) .{ }^{14}$ Then the common slope $q^{*}$ of the zero-profit line $00^{\prime}$ and of the consumer's indifference curve in $\left(\alpha^{*}, \beta^{*}\right)$ is strictly greater than the premium-benefit ratio $\beta^{*} / \alpha^{*}$ : As insurance coverage is increased, moral hazard drives the accident probability up, and an increase in the premium-benefit ratio is needed to avoid losses. Then one has:

Proposition 3: If $p(\cdot)$ is strictly convex on the set $E=[\underline{e}, \infty)$ and $e^{*}>\underline{e}, w-\beta^{*}>w-d+\alpha^{*}$, then there is no MCE with potential entry in which all firms share information about their customers.

This proposition is proved by contradiction. Suppose that $(\dot{G}, \bar{\pi})$ is an MCE with potential entry in which all firms share information about their customers. Then the consumer's choice $(e, \bar{c}) \in \emptyset(G)$ must yield the "second best " allocation $\left(e^{*}, \alpha^{*}, \beta^{*}\right)$, i.e. one must have $e=e^{*}, \sum_{i} \bar{\alpha}_{i}=\alpha^{*}, \sum_{i} \bar{\beta}_{i}=\beta^{*}$. Otherwise a potential entrant could make a strictly positive profit by offering the contract $\left(\left(\alpha^{*}-\varepsilon, \beta^{*}\right), 0\right)$ on condition that no other insurance is purchased. Since all other firms share information about their customers ${ }^{15}$, the entrant would be sure to have only customers without supplementary insurance. Moreover, for $\varepsilon$ sufficiently small, consumers would strictly prefer the triple $\left(e^{*}, \alpha^{*}-\varepsilon, \beta^{*}\right)$ over $(e, \bar{c})$ and buy insurance from the entrant who would therefore make a profit. To rule out this type of entry, one must have $e=e^{*}, \sum_{i} \bar{\alpha}_{i}=\alpha^{*}$, and $\sum_{i} \bar{\beta}_{i}=\beta^{*}$.

But now Figure 2 shows that the "second best" allocation cannot correspond to an MCE with potential entry either. For consider an entrant with the unconditional supplementary offer $\left(\left(\alpha_{1}-\alpha^{*}, \beta_{1}-\beta^{*}\right), 0\right)$, which brings the consumer to the point $\left(\alpha_{1}, \beta_{1}\right)$ in figure 2 . For specificity, assume that the premium-benefit ratio on this supplementary contract is halfway between the slope of the zero-profit line, $q^{*}$, and the ratio $\beta^{*} / \alpha^{*}$, i.e. $\beta_{1}-\beta^{*} \frac{q^{*}+\beta^{*} / \alpha^{*}}{2}\left(\alpha_{1}-\alpha^{*}\right)$. For $\left(\alpha_{1}-\alpha^{*}\right)$ sufficiently small, the consumer will definitely wish to buy this supplement, provided it is kept and has no effect on the feasible contract combinations from other firms. Moreover, for $\left(\alpha_{1}-\alpha^{*}\right)$ sufficiently small, the supplement has only a small effect on the consumer's effort level, so that the break-even premiumbenefit ratio remains close to $\beta^{*} / \alpha^{*}$. Since $\left(\beta_{1}-\beta^{*}\right) /\left(\alpha_{1}-\alpha^{*}\right)$ is significantly greater than $\beta^{*} / \alpha^{*}$, the supplement will make a strictly positive profit, even though all contracts together will now make a loss. This completes the proof of Proposition 3.

The preceding argument shows how firms may wish to conceal their customers' identities in order to preclude adverse reactions from other firms. This secrecy affects the

${ }^{14}$ If $p^{\prime \prime}>0$, this follows directly from the first order condition for $e$, $p^{\prime}(e)[u(w-\beta)-u(w-d+\alpha)] \leqslant 1$, with inequality only if $e=\underline{e}$.

is Since the entrant sets $s=0$, purchase of his contract has no effect on the available offers from other firms. 
market in two ways. On the one hand, entry with a supplementary insurance offer involving secrecy may destabilize a strategy array that would otherwise be an equilibrium. This possibility is illustrated by the proof of Proposition 3. On the other hand, the availability of inactive contract offers involving secrecy may discourage entry because an entrant has no control over supplementary insurance purchases. In Proposition 2 this effect was strong enough to support a perfect equilibrium with positive profits.

Which of these two effects prevails depends on the exogenous data of the model, in particular on the functions $u$ and $p$. A general analysis of this problem is quite difficult and beyond the scope of these comments. However, if effort is a discrete variable which can take on only two values, the following proposition and its proof provide a complete characterization of perfect equilibria.

Proposition 4: A perfect MCE with potential entry exists whenever $e$ can take on only two values.

The proof of Proposition 4 is based on the geometry of the zero-profit line when effort takes on only two values. Let $e, \bar{e}$ be the two effort levels, et $\bar{p}=p(e), p=p(\bar{e})$ be the corresponding accident probabilities, and let $\bar{q}=\bar{p} /(1-\bar{p}), q=p /(1-\underline{p})$ be the corresponding break-even premium-benefit ratios. From Professor Stiglitz's analysis (see, e.g., his Figure 5a), we know that the zero-profit line lies between the rays through the origin with slopes $\bar{q}$ and $q$. More precisely, for large values of $\alpha$ and $\beta$, the agent chooses the low effort level $e$ because he does not care very much whether an accident occurs. The accident probability then is $\bar{p}$, and zero profits require a premium-benefit ratio $\bar{q}$ (which is infinite if $\bar{p}=1$ ). If the difference in accident probabilities, $\bar{p}-\underline{p}$, is sufficiently large relative to the difference in effort levels, $\bar{e}-\underline{e}$, then for small values of $\alpha$ and $\beta$, the agent prefers the high effort level $\bar{e}$, because with little insurance he cares a lot whether an accident occurs. The accident probability then is $p$, and zero profits require a premium-benefit $q$. For intermediate values of $\alpha$ and $\beta$, the agent may be indifferent between $e$ and $\bar{e}$ and choose either with positive probability. In this case the break-even premium-benefit ratio lies strictly between $\bar{q}$ and $q$. In general then, the zero-profit line has the shape of the line 00' in Figure 3-5.

First assume that $\bar{q}<\infty$. For any point $(\underline{\alpha}, \underline{\beta})$ on the ray of slope $\underline{q}$ through the origin, consider the new ray of slope $\bar{q}$ that starts at $(\underline{\alpha}, \underline{\beta}),\{(\alpha, \beta) \mid(\alpha, \beta)=(\underline{\alpha}, \underline{\beta}) \delta(1, \bar{q}), \delta \geqslant 0\}$. Let $\hat{\lambda}(\alpha, \beta)$ be the set of triples $(\hat{e}, \hat{\alpha}, \hat{\beta})$ that maximize expected utility $(1-p(\hat{e})) u(w-\hat{\beta})+p(\hat{e}) u(w-d+\hat{\alpha})-\hat{e}$ subject to the constraint that $(\hat{\alpha}, \hat{\beta})$ belong to the $\operatorname{ray}\{(\alpha, \beta) \mid(\alpha, \beta)=(\underline{\alpha}, \underline{\beta})+\delta(1, \bar{q}), \delta \geqslant 0\}$. Observe that for $\hat{e}=\underline{e}$, the accident probability is $\bar{p}$. Then the premium-benefit ratio $\bar{q}$ is fair, and the agent demands full insurance. Therefore $(\hat{e}, \hat{\alpha}, \hat{\beta}) \in \hat{\lambda}(\underline{\alpha}, \underline{\beta})$ for some $(\bar{\alpha}, \bar{\beta})$ implies :

$\hat{e}=\bar{e}$ if and only if $w-\hat{\beta}=w-d+\hat{\alpha}$, i.e.

$\hat{\alpha}=\frac{1}{1+\bar{q}}[d+\bar{q} \underline{\alpha}-\underline{\beta}], \hat{\beta}=\frac{\bar{q}}{1+\bar{q}}[d-\bar{q} \underline{\alpha}+\underline{\beta}]$

Let $(\bar{\alpha}, \bar{\beta})$ be the unique point on the ray of slope $q$ through the origin at which the agent is indifferent between the effort levels $e$ and $\bar{e}$ (see Figures 3 and 4 ). At $(\bar{\alpha}, \bar{\beta})$, the agent is willing to choose the low effort level $\underline{e}$. At all points $(\underline{\alpha}, \underline{\beta})$ to the right of $(\bar{\alpha}, \bar{\beta})$, the agent strictly prefers $e$ to $\bar{e}$. Therefore he regards additional insurance at the premium-benefit ratio $\bar{q}$ as fair, and we have 


$$
\hat{\lambda}(\underline{\alpha}, \underline{\beta})=\left\{\left(\underline{e}, \frac{d+\bar{q} \underline{\alpha}-\underline{\beta}}{1+\bar{q}}, \frac{\bar{q}(d-\bar{q} \underline{\alpha}+\underline{\beta})}{1+\bar{q}}\right)\right\}
$$

for $(\underline{\alpha}, \underline{\beta})$ equal to or to the right of $(\bar{\alpha}, \bar{\beta})$.

Now there are two possibilities. Either (i) : (5) holds for all $(\underline{\alpha}, \underline{\beta})$, or (ii) : (5) fails for some $(\alpha, \underline{\beta})$ on the ray of slope $q$ through the origin. First assume (i). Then a perfect equilibrium with potential entry is given by the strategies:

$$
\begin{array}{ll}
g_{i}(\gamma)=\left(\frac{1}{k}\left(\frac{\alpha}{1+\bar{q}}, \frac{\bar{q} \delta}{1+\bar{q}}\right), 0\right) & \text { for all } \gamma \in \mathbb{R}_{+}^{2}, \\
i=2 k, k=1,2, \ldots, & \text { for all } \gamma \in \mathbb{R}_{+}^{2}, \\
g_{i}(\gamma)=((0,0), 0) & \\
i=2 k-1, k=1,2, \ldots . &
\end{array}
$$

In the equilibrium, the consumer buys full insurance from firm 1, without supplementary insurance. The equilibrium effort level is $\underline{e}$, and all firms make zero profits.

Alternatively assume (ii), and let $\left(\alpha_{0}, \underline{\beta}_{0}\right)$ be such that $\alpha_{0}$ is the least upper bound of the set of $\alpha$ such that (5) fails for $(\alpha, \underline{\beta}=q \alpha)$ (Figures 3,4), i.e. $\left(\underline{\alpha}_{0}, \underline{\beta}_{0}\right)$ is the rightmost discontinuity point of the relation $\hat{\lambda}$. By a similar argument as in Lemma 2.1, we may assert the existence of $\left(e_{0}, \alpha_{0}, \beta_{0}\right) \in \hat{\lambda}\left(\underline{\alpha}_{0}, \underline{\beta}_{0}\right)$, such that $e_{0}=\bar{e}$ and $w-\beta_{0}>w-d+\alpha_{0} ;$ the triple $\left(e_{0}, \alpha_{0}, \beta_{0}\right)$ involves partial insurance and a high effort level.

With this specification of $\left(\alpha_{0}, \beta_{0}\right)$, a perfect equilibrium with potential entry is again given by the strategies $(4 a)-(4 c)$ of Proposition 2 . Given these strategies, the consumer buys exactly one of the contracts $\left(\left(\alpha_{0}, \beta_{0}\right), 1\right)$. By construction, he cannot supplement this contract with another identical contract, and he does not care to supplement it with contracts of the form $(\delta(1, \bar{q}), 0)$. Again by construction, we have $\beta_{0} \geqslant q \alpha_{0}$ and $e_{0}=\bar{e}$, so that the firm selling the contract $\left(\left(\alpha_{0}, \beta_{0}\right), 1\right)$ will at least break even.

Finally, the same argument as in the proof of Proposition 2 shows that no firm can do better by changing its strategy. To do better, it would have to bring the agent to a point $\left(\alpha_{1}, \beta_{1}\right)$ to the right of the ray $\left\{(\alpha, \beta) \mid(\alpha, \beta)=\left(\underline{\alpha}_{0}, \underline{\beta}_{0}\right)+\delta(1, \bar{q}), \delta \geqslant 0\right\}$. However, by definition of $\left(\alpha_{0}, \beta_{0}\right)$, the consumer will then buy supplementary contracts at the premium-benefit ratio $\bar{q}$ up to the point $\left(\alpha_{2}, \beta_{2}\right)$ where he has full insurance. His effort level then is $e$, and all firms asking a premium-benefit ratio less than $\bar{q}$ will make a loss. Hence an offer such as $\left(\alpha_{1}, \beta_{1}\right)$ cannot improve any firm's position, and the strategies $(4 a)-(4 c)$ are a perfect equilibrium.

It remains to deal with the case $\bar{q}=\infty$, or $p(\underline{e})=1$. As before let $(\bar{\alpha}, \bar{\beta})$ be the point on the ray of slope $q$ through the origin at which the agent is indifferent between the effort levels $\underline{e}$ and $\bar{e}$ (Figure 5). A perfect equilibrium with potential entry is given by the strategies :

$$
\begin{aligned}
& i=2 k, \quad k=1,2, \ldots, \\
& g_{i}(\gamma)=((0,0), 0) \\
& i=2 k-1, \quad k=1,2, \ldots .
\end{aligned}
$$

In the equilibrium, the consumer buys exactly one of the contracts $((\bar{\alpha}, \bar{\beta}), 1)$ and chooses the effort level $\bar{e}$. Since $\bar{\beta}=q \bar{\alpha}$, the insurance company offering this contract makes exactly zero profits. 
One easily verifies that in this case the allocation $(\bar{e}, \bar{\alpha}, \bar{\beta})$ coincides with the "second best" allocation $\left(e^{*}, \alpha^{*}, \beta^{*}\right)$ (see Figure 5). Any firm that wishes to improve its position must therefore try to attract the consumer by enabling him to reach a point $\left(\alpha_{1}, \beta_{1}\right)$ to the right of the zero-profit line. At this point all insurance companies together make a loss. Since the insurance company in question does not want to incur a loss, the point $\left(\alpha_{1}, \beta_{1}\right)$ must be reached by a secret supplement, $\left(\left(\alpha_{1}-\bar{\alpha}, \beta_{1}-\bar{\beta}\right), 0\right)$ to the original contract $((\bar{\alpha}, \bar{\beta}), 1)$. By definition of $(\bar{\alpha}, \bar{\beta})$ it follows that at $\left(\alpha_{1}, \beta_{1}\right)$ the consumer chooses the low effort level $e$ entailing the accident probability $\bar{p}=1$. But then the supplement $\left(\left(\alpha_{1}-\bar{\alpha}, \beta_{1}-\bar{\beta}\right), 0\right)$ must make a loss. It follows that the strategies $(7 \mathrm{a})-(7 \mathrm{~b})$ are indeed a perfect MCE with potential entry. This completes the proof of Proposition 4 .

Proposition 4 provides a new perspective on Professor Stiglitz's discussion of the existence of equilibrium in the absence of communication. At least for the case of two effort levels, there always exists an equilibrium with communication. The question then is why the absence of communication might destroy the existence of equilibrium.

In the equilibria of Proposition 4, communication makes it possible to have both, quantity rationing and competition. Competition is such that none of the firms offering the contract $\left(\left(\alpha_{0}, \beta_{0}\right), 1\right)$ can afford to offer the consumer less favourable insurance terms ; as in Bertrand oligopoly, the consumer can always threaten to go elsewhere and buy the contract $\left(\left(\alpha_{0}, \beta_{0}\right), 1\right)$ from another firm. At the same time, communication among firms makes sure that the consumer buys no more than one of the contracts $\left(\left(\alpha_{0}, \beta_{0}\right), 1\right)$ though he certainly would like to have more insurance at the premium-benefit ratio $\beta_{0} / \alpha_{0}$.

Without communication among firms, this combination of quantity control and competition is infeasible. To ration the consumer's purchases below what he would like to buy at the given premium-benefit ratio, one needs a limit on the total amount of insurance that all insurance companies together offer at this premium-benefit ratio. But then, the consumer cannot go elsewhere if some company raises its premium or reduces its coverage. Consequently, individual insurance companies will have some monopoly power of their customers.

Because of this monopoly power, it is generally impossible to attain the equilibrium allocation $\left(\bar{e}, \alpha_{0}, \beta_{0}\right)$ of Proposition 4 in an MCE without communication. For suppose that we had an MCE without communication in which the consumer's aggregate insurance purchase is $\left(\alpha_{0}, \beta_{0}\right)$. For at least one firm $i$, the consumer's purchase $\left(\bar{\alpha}_{i}, \bar{\beta}_{i}\right)$ from firm $i$ satisfies $0<\bar{\beta}_{i} / \bar{\alpha}_{i} \leqslant \beta_{0} / \alpha_{0}$. We know that the slope of the consumer's indifference curve at $\left(\alpha_{0}, \beta_{0}\right)$ is strictly greater than $\beta_{0} / \alpha_{0}$ (see Proposition 1 , figures 3,4 ). This implies that (i) no further contract at the premium-benefit ratio $\beta_{0} / \alpha_{0}$ is available, hence (ii) if firm $i$ were to withdraw the offer $\left(\left(\bar{\alpha}_{i}, \bar{\beta}_{i}\right), 0\right)$, the consumer would be strictly worse off because he has no replacement for firm $i$. But then firm $i$ can increase its profits by offering a contract $\left(\left(\bar{\alpha}_{i}-\delta, \bar{\beta}_{i}+\varepsilon\right), 0\right)$ rather than $\left(\left(\bar{\alpha}_{i}, \bar{\beta}_{i}\right), 0\right)$, where $\delta>0, \varepsilon>0$ are chosen so that the consumer is hereby willing to accept the offer $\left(\left(\bar{\alpha}_{i}-\delta, \bar{\beta}_{i}+\varepsilon\right), 0\right)$ rather than go without a contract from firm $i{ }^{16}$ This contradicts the assumption that $\left(\left(\bar{\alpha}_{i}, \bar{\beta}_{i}\right), 0\right)$ is an equilibrium contract offer.

16 The above argument makes essential use of the discreteness of firms. If we had a continuum of insurance companies, the monopoly power of individual firms would disappear. However, Proposition 5 below suggests that this is a mathematical artefact which has no economic interpretation on the limit of systems with large finite numbers of active firms. 
For example, consider a constellation with a large number of firms offering arbitrary amounts of insurance at the high premium-benefit ratio $\bar{q}$ and exactly one firm, say firm 1 , offering a limited amount of insurance at a lower premium-benefit ratio. Starting from the contract $\left(\left(\alpha_{0}, \beta_{0}\right), 1\right)$, firm 1 will try to raise its profits by lowering its benefit and raising its premium subject to the two constraints that (i) the consumer is still willing to buy from firm 1, and (ii) the consumer does not wish to obtain full insurance by supplementing firm I's contract with other contracts at the premium-benefit ratio $\bar{q}$. The role of these considerations is shown in Figure 6. The line $\mathrm{AA}^{\prime}$ is the locus of all points at which the agent is indifferent between staying where he is and buying supplementary contracts up to full insurance at the premium-benefit ratio $\bar{q}$. The line $\mathrm{BB}^{\prime}$ is the indifference curve corresponding to the highest utility level that can be reached without firm 1 . Firm 1 will choose insurance terms $(\alpha, \beta)$ in the region below both $\mathrm{AA}^{\prime}$ and $\mathrm{BB}^{\prime}$ so as to maximize $(1-\underline{p}) \beta-\underline{p} \alpha$. It will therefore offer the contract $\left(\left(\alpha_{1}, \beta_{1}\right), 0\right)$ that corresponds to the intersection of $\mathrm{AA}^{\prime}$ and $\mathrm{BB}^{\prime}$.

The question now is whether this constellation in which consumers buy the contract $\left(\left(\alpha_{1}, \beta_{1}\right), 0\right)$ from firm 1 can be an equilibrium without communication. At first sight, there should be room for entry, say with the contract $\left(\left(\alpha_{0}, \beta_{0}\right), 0\right)$, which the consumer prefers to $\left(\left(\alpha_{1}, \beta_{1}\right), 0\right)$. However, if firm 2 enters the market with the contract offer $\left(\left(\alpha_{0}, \beta_{0}\right), 0\right)$, the consumer may decide to buy both $\left(\left(\alpha_{0}, \beta_{0}\right), 0\right)$ and $\left(\left(\alpha_{1}, \beta_{1}\right), 0\right)$ and to supplement these contracts at the premium-benefit ratio $\bar{q}$ to go up to full insurance. Then the accident probability is $\bar{p}$, and the new contract $\left(\left(\alpha_{0}, \beta_{0}\right), 0\right)$ makes a loss.

In general, the vulnerability of contracts such as $\left(\left(\alpha_{1}, \beta_{1}\right), 0\right)$ to entry seems to depend on the exogenous data of the system, in particular on the consumer's indifference curves. Under the additional assumption of constant absolute risk aversion, we obtain the following result, which is proved in the appendix :

Proposition 5 : Let $u(c)=-\exp (-c)$ and assume that $e$ can take on only the two values $\underline{\underline{e}}$ and $\bar{e}$. If $(1-\underline{p})(\exp (d)-1)>(\bar{e}-\underline{e}) \exp (w)$ there exists $\varepsilon>0$, such that for $\overline{\bar{p}}>1-\varepsilon$, the following are true :

a : The high effort level $\bar{e}$ with zero insurance is strictly preferred to the low effort level $\underline{e}$ with full insurance at the rate $\bar{q}=\bar{p} /(1-\bar{p})$.

$\mathrm{b}$ : There exists a price equilibrium with positive effort.

c: For every integer $n$, there exist insurance terms $\left(\alpha_{n}, \beta_{n}\right)$, such that the following strategy array yields a non-price MCE without communication :

$$
\begin{array}{ll}
g_{i}(\gamma)=\left(\frac{1}{n}\left(\alpha_{n}, \beta_{n}\right), 0\right) & \text { for all } \gamma \in \mathbb{R}_{+}^{2}, \\
i=1,2, \ldots n, & \\
g_{n+1}(\gamma)=((0,0), 0) & \text { for all } \gamma \in \mathbb{R}_{+}^{2}, \\
g_{i}(\gamma)=\left(\frac{1}{i}(1, \bar{q}), 0\right) & \text { for all } \gamma \in \mathbb{R}_{+}^{2}, \\
i=n+2, n+3, \ldots . &
\end{array}
$$

$\mathrm{d}$ : The sequence $\left\{\alpha_{n}, \beta_{n}\right\}_{n=1}^{\infty}$, is bounded away from the equilibrium insurance terms $\left(\alpha_{0}, \beta_{0}\right)$ of the perfect MCE with communication in Proposition 4.

e: For $n$ sufficiently large, the allocation $\left(\underline{e}, \alpha_{n}, \beta_{n}\right)$ is strictly preferred to the price equilibrium allocation in (b). 
In the first place, Proposition 5 shows that even without communication we may have equilibria that are not price quilibria. In such equilibria, quantity rationing occurs because only $n$ insurance companies offer insurance at a premium-benefit ratio less than $\bar{q}$. As $n$ increases, the consumer's utility in these equilibria goes up, presumably because there is more competition among firms. For sufficiently large $n$, the non-price equilibria are preferred to the price equilibria with positive profits, but the consumer's utility never even comes close to his utility in the perfect MCE with communication. This result provides striking evidence for the proposition that the absence of communication reduces the degree of competition that is compatible with market equilibrium. ${ }^{17}$

Secondly, Proposition 5 shows that Professor Stiglitz's analysis of the existence of equilibrium is inconclusive because he restricts himself to price equilibria. Specifically, we have obtained a non-price equilibrium for the very constellation of Figure 8 in his paper for which he claims nonexistence of a zero profit price equilibrium. ${ }^{18}$ The constellation of Figure 10b, for which Professor Stiglitz also claims the nonexistence of a price equilibrium, does not arise under constant absolute risk aversion; it is therefore not covered by Proposition 5.

Based on some diagrammatic exercises, I conjecture (i) that for some cases, an MCE without communication does not exist, but that (ii) the constellation of Figure $10 \mathrm{~b}$ in Professor Stiglitz's paper is not sufficient for this nonexistence result. At this point, I have no explicit examples for either conjecture, and the question of existence remains open. ${ }^{19}$

\section{Concluding remarks}

The preceding notes leave a number of questions open. In addition to the general existence problem for equilibria without communication, it will be necessary to reconsider the existence problem for MCE's with communication when there are more than two effort

${ }^{17}$ Statements (d) and (e) of Proposition 5 hold quite generally and do not require the assumption of constant absolute risk aversion.

${ }^{18}$ In passing, we note that the same constellation also gives rise to a price equilibrium with positive effort which involves either zero insurance or positive profits. In general, $\left(e, \frac{d}{1+\bar{q}}, \frac{\bar{q} d}{1+\bar{q}}\right) \in \lambda(0, \bar{q})$ implies either the constellation of his Figure 10a or the constellation of his Figure 10b. The constellation of his Figure $10 \mathrm{a}$ gives rise to a price equilibrium with positive effort. This equilibrium involves zero profits if and only if the points $F$ and 0 in Figure $10 \mathrm{~b}$ coincide, i.e. if and only if $(\bar{e}, 0,0) \in \lambda\left(0, q^{*}\right)$, where $q^{*}$ is the premium-benefit ratio at which the agent jumps from underinsurance with maximum effort to overinsurance with minimum effort. Specifically, the price equilibrium with positive effort involves zero insurance if $d$ is small and positive profits if $d$ is large. In general then, the constellation of his Figure $10 \mathrm{~b}$ is necessary and sufficient for the nonexistence of a price equilibrium.

${ }^{19}$ Since writing the above, 1 have constructed examples confirming both conjectures. In particular, let $u(c)=c^{\gamma} / \gamma, \gamma \in(0,1)$. For $\bar{q}$ and $\bar{e}-e$ large enough, no MCE without communication will exist. On the other hand, for $q$ and $e-e$ small enough, one has a nonprice equilibrium with $n$ firms, provided $n$ is sufficiently large. At the same time, the demand relation $\lambda$ gives rise to the constellation of Figure 10b in Professor Stiglitz's lecture. Thus, neither of Professor Stiglitz's conditions is in fact sufficient for the nonexistence of equilibrium. I will present these results in a forthcoming working paper. 
levels. It is relatively easy to extend the construction of Proposition 4 to any finite number of effort levels. However, the resulting equilibria will typically not be perfect, and, in general, perfect equilibria will not exist. If effort is a continuous variable, I conjecture that in some cases no equilibrium with or without communication will exist.

At another level, one must question the concept of "secret" contracts that underlies Professor Stiglitz's lecture as well as these comments. Specifically, one must ask why an insurance company would fulfil its obligations under such a contract. If the insurance company refuses to pay in case of an accident, the consumer must go to court to obtain his benefit. Through the litigation, the contract would automatically become public, and the consumer would forfeit all claims on those contracts that have exclusivity requirements. If the consumer foresees this problem of moral hazard on the side of the insurance company, he will simply not accept a secret contract. Secret contracts can only be viable if insurance companies will always fulfil their obligations because they fear the adverse effects of litigation on their reputations.

Empirically, one observes that insurance companies do not usually use quantity constraints to affect their customers' efforts at accident prevention. Instead, they rely on intertemporal incentive devices, e.g. experience ratings, whereby tomorrow's premium depends on today's claims for damages. Like quantity constraints in a static model, such intertemporal incentive devices leave the consumer with some residual accident risk, this time some risk concerning future insurance premia. The main difference between intertemporal incentive devices and quantity constraints concerns the timing of this residual risk. Quantity constraints concern payments at the time of an accident ; premium risk concerns payments in later periods. The question is whether this difference in timing explains the widespread preference for experience ratings over direct quantity constraints. To answer this question, one would need an explicit model of insurance and capital markets in several periods.

\section{Appendix}

Like Professor Stiglitz, I assume that the utility function $u$ is differentiable, strictly increasing, and strictly concave. The function $p$ is continuous and strictly decreasing on the interval $[\underline{e}, \infty)$. Note that the agent's income is state-independent if and only if $\alpha=d-\beta$.

Lemma A.l: For $v<d q$, let $(e, \alpha, \beta) \in \lambda(v, q)$. Then $\alpha \gtreqless d-\beta$ as $q \lesseqgtr p(e) /(1-p(e))$. Moreover $q \leqslant p(e) /(1-p(e))$ implies $e=\underline{e}$ and $p(e)=\vec{p}$.

Proof: Given the constraint $\beta=q \alpha-v$, the first order condition for $\alpha$ and $\beta$ is :

$$
-q(1-p(e)) u^{\prime}(w-\beta)+p(e) u^{\prime}(w-d+\alpha) \leqslant 0,
$$

with equality if $\beta>0$. For $\beta=0, \alpha=v / q<d$, the strict concavity of $u$ implies $q(1-p(e)) \geq p(e)$. For $\beta>0$, the strict concavity of $u$ implies $w-\beta \leqq w-d+\alpha$ as $q(1-p(e)) \lesseqgtr p(e)$. The first statement of the lemma follows immediately. For $q \leqslant p(e) /(1-p(e))$ then one has $w-e+\alpha \geqslant w-\beta$, and effort must be at its minimum level $\underline{e}$.

Q.E.D. 
Proof of Proposition 1: Let $(g, \pi)$ be an MCE with potential entry, let $(e, c) \in \emptyset(G)$ be the equilibrium choice of the consumer, i.e. $\bar{\pi}_{i}=\bar{\pi}_{i}(e, \bar{c})$ for all $i$, and let $q=\sum_{i} \bar{\beta}_{i} / \sum_{i} \bar{\alpha}_{i}$ be the aggregate premium-benefit ratio.

One must have $\bar{\beta}_{i}-\bar{q} \bar{\alpha}_{i} \leqslant 0$ for all $i$. If one had $\bar{\beta}_{i_{0}}-\bar{q} \bar{\alpha}_{i_{0}}>0$ for some firm $i_{0}$, a potential entrant could displace firm $i_{0}$ with the offer $\left(\left(\bar{\alpha}_{i_{0}},\left(\bar{\beta}_{i_{0}}+\bar{q} \bar{\alpha}_{i_{0}}\right) / 2, s_{i_{0}}\right)\right.$. Since the accident probability $p(e)$ cannot exceed $\bar{p}$, this contract would make strictly positive profits in contradiction to the condition that entry is not profitable.

It follows that $q=\bar{q}$ implies $\bar{\beta}_{i}-\bar{q} \bar{\alpha}_{i}=0$ for all $i$. Perfectness of the MCE then follows trivially because contracts with the premium-benefit ratio $\vec{q}$ can never make a loss.

The converse half of the proposition is proved by contradiction. Suppose that $(G, \bar{\pi})$ is perfect, and that $q<\bar{q}$. Since profits are nonnegative, the accident probability $p(e)$ must be strictly less than $\bar{p}$, or $e>\underline{e}$. Then Lemma A.1 implies $q>p(e) /(1-p(e))$, or:

$$
(1-p(e)) \sum_{i} \bar{\beta}_{i}-p(e) \sum_{i} \bar{\alpha}_{i}>0 .
$$

For a potential entrant $j$, consider the contract offers $\left(\gamma_{j}^{n}, 1\right), \gamma_{j}^{n}=\left(\sum_{i} \bar{\alpha}_{i}, \sum_{i} \bar{\beta}_{i}-1 / n\right)$, $n=1,2, \ldots$. Clearly $\gamma_{j}^{n} \in F(G)$ for all $n$. By perfectness of $(G, \bar{\pi})$, there exist $\left(e^{n}, \hat{c}^{n}\right) \in \emptyset\left(\gamma_{j}^{n}, G\right)$, such that :

$$
\left(1-p\left(e^{n}\right)\right) \sum_{i} \hat{\beta}_{i}^{n}-p\left(e^{n}\right) \sum_{i} \hat{\alpha}_{i}^{n} \leqslant 0 .
$$

Since $(G, \bar{\pi})$ is an MCE, entry with the unconditional offer $\left(\gamma_{j}^{n}, 1\right)$ is unprofitable, i.e.

$$
\left(1-p\left(e^{n}\right)\right)\left(\sum_{i} \bar{\beta}_{i}-1 / n\right)-p\left(e^{n}\right) \sum_{i} \bar{\alpha}_{i} \leqslant 0 \text {. }
$$

Further, for all $n$, the triple $\left(e^{n}, \sum_{i}\left(\bar{\alpha}_{i}+\hat{\alpha}_{i}^{n}\right), \sum_{i}\left(\bar{\beta}_{i}+\hat{\beta}_{i}^{n}\right)-\frac{1}{n}\right)$ is preferred to $\left(e, \sum_{i} \bar{\alpha}_{i}, \sum_{i} \bar{\beta}_{i}-\frac{1}{n}\right)$ and hence to $\left(e, \sum_{i} \bar{\alpha}_{i} \sum_{i} \bar{\beta}_{i}\right)$. Since $(G, \bar{\pi})$ is a price equilibrium, it follows that :

$$
\begin{aligned}
& \left(1-p\left(e^{n}\right)\right) u\left(w-\sum_{i}\left(\bar{\beta}_{i}+\hat{\beta}_{i}^{n}\right)+\frac{1}{n}\right)+p\left(e^{n}\right) u\left(w-d+\sum_{i}\left(\bar{\alpha}_{i}+\hat{\alpha}_{i}^{n}\right)\right)-e^{n} \\
& \geqslant\left(1-p\left(e_{0}\right)\right) u\left(w-\beta_{0}\right)+p\left(e_{0}\right) u\left(w-d+\alpha_{0}\right)-e_{0} \\
& \text { for all } e_{0} \geqslant \underline{e}, \beta_{0}=q \alpha_{0} \geqslant 0 .
\end{aligned}
$$

Taking a subsequence if necessary let $p\left(e^{n}\right) \rightarrow p^{\infty}$ and $e^{n} \rightarrow e^{\infty}=p^{-1}\left(p^{\infty}\right)$. Let $\hat{q}^{n}=\sum_{i} \hat{\beta}_{i}^{n} / \sum_{i} \hat{\alpha}_{i}^{n}$ be the aggregate premium-benefit ratio for the supplement $\hat{c}^{n}$. (A.1) and (A.2) imply $\lim _{n \rightarrow \infty} \inf \hat{q}^{n} \geqslant q$. Hence either $\lim _{n \rightarrow \infty} \sup \hat{q}^{n}>q$ or $\lim _{n \rightarrow \infty} \hat{q}^{n}=q$.

Case $1: \limsup _{n \rightarrow \infty} \hat{q}^{n}>q$.

There exist $\varepsilon>0$ and a subsequence $\left\{n^{k}\right\}_{k=1}^{\infty}$, such that $\hat{q}^{n^{k}} \geqslant q+\varepsilon$ for all $k$. For $k=1,2, \ldots$, observe that (A.4) with $e_{0}=e^{n^{k}}, \quad \alpha_{0}=\sum\left(\bar{\alpha}_{i}+\hat{\alpha}_{i}^{n^{k}}\right)$, and $\beta_{0}=q \alpha_{0} \leqslant \sum_{i}\left(\bar{\beta}_{i}+\hat{\beta}_{i}^{n^{k}}\right)-\varepsilon \sum_{i} \hat{\alpha}_{i}^{n^{k}}$ implies $\varepsilon \sum_{i} \hat{\alpha}_{i}^{n^{k}} \leqslant 1 / n^{k}$, hence $\lim _{k \rightarrow \infty} \sum_{i} \hat{\alpha}_{i}^{n^{k}}=\lim _{n \rightarrow \infty} \sum_{i} \hat{\beta}_{i}^{n^{k}}=0$.

Taking limits as $k \rightarrow \infty$ in both (A.4) and (A.3), one then has $\left(e^{\infty}, \sum_{i} \bar{\alpha}_{i}, \sum_{i} \bar{\beta}_{i}\right) \in \lambda(0, q)$ and $\left(1-p^{\infty}\right) \sum \bar{\beta}_{i}-p^{\infty} \sum_{i} \bar{\alpha}_{i} \leqslant 0$, or $\left(1-p^{\infty}\right) q \leqslant p^{\infty}$. Therefore Lemma A.1 implies $\sum_{i} \bar{\alpha}_{i} \geqslant d-\sum_{i} \bar{\beta}_{i}$, and, again by Lemma A.1, $\left(e, \sum_{i} \bar{\alpha}_{i}, \sum_{i} \bar{\beta}_{i}\right) \in \lambda(0, q)$ implies $\stackrel{i}{q} \leqslant p(e) /(1-p(e))$, hence $(1-p(e)) \sum_{i} \bar{\beta}_{i}-p(e) \sum_{i} \bar{\alpha}_{i} \leqslant 0$, contrary to (A.l). 
Case $2: \lim _{n \rightarrow \infty} \hat{q}^{n}=q$.

Now (A.2) and the assumption $q<\bar{q}$ imply $e^{n}>\underline{e}$ for all but finitely many $n$. Therefore the same argument as in the proof of Lemma A.1 implies $\sum_{i}\left(\bar{\alpha}_{i}+\hat{\alpha}_{i}^{n}\right)+\sum_{i}\left(\bar{\beta}_{i}+\hat{\beta}_{i}^{n}\right) \leqslant d$ for all but finitely many $n$. Taking a subsequence if necessary, one may therefore suppose that $\sum_{i} \hat{\alpha}_{i}^{n} \rightarrow \hat{\alpha}$ and $\sum_{i} \hat{\beta}_{i}^{n} \rightarrow \hat{\beta}$. Clearly $\hat{\beta}=q \hat{\alpha}$. Taking limits as $n \rightarrow \infty$ in (A.4), A.2), and '(A.3), one has $\left(e^{\infty}, \sum \bar{\alpha}_{i}+\hat{\alpha} \mathrm{K} \sum \bar{\beta}_{i}+\hat{\beta}\right) \in \lambda(0, q), \quad\left(1-p^{\infty}\right) \hat{\beta}-p^{\infty} \hat{\alpha} \geqslant 0$, and $\left(1-p^{\infty}\right) \sum_{i} \bar{\beta}_{i}-p^{\infty} \sum_{i} \bar{\alpha}_{i} \leqslant 0$, hence $\left(1-p^{\infty}\right) q=p^{\infty}$. Therefore Lemma A.1 implies $e^{\infty}=e, p^{\infty}=\bar{p}$, and hence $q=\bar{q}$, contrary to the assumption that $q<\bar{q}$. Therefore the assumption that $(G, \pi)$ is a perfect price equilibrium with potential entry and $q<\bar{q}$ is false. This completes the proof of Proposition 1.

Q.E.D.

Lemma A.2: The relation $(v, q) \rightarrow \lambda(v, q)$ is upper hemi-continuous at every $(v, q)$ such that $v \geqslant 0$ and $q>0$.

Proof: Let $\bar{\lambda}(v, q)$ be the set of maximizers of the continuous function $(1-p(e)) u(w-\beta)+p(e) u(w-d+\alpha)-e$ on the compact set $\{(e, \alpha, \beta) \mid \underline{e} \leqslant e \leqslant \underline{e}+u(w+(w+v) / q)-u(w-d), \quad 0 \leqslant \beta=q \alpha-v \leqslant w\}$. By the maximum theorem (see e.g. Hildenbrand [1974], p. $29 \mathrm{f}$ ). the relation $\bar{\lambda}$ is upper hemi-continuous at $v \geqslant 0, q>0$. Therefore it suffices to show that $\lambda(v, q)=\bar{\lambda}(v, q)$ for all $v \geqslant 0$ and $q>0$. For $\left(e_{0}, \alpha_{0}, \beta_{0}\right)$ with $e_{0}>\underline{e}+u(w+(w+v) / q)-u(w-d)$ and $0 \leqslant \beta_{0}=q_{0}-v \leqslant w$, one has:

$$
\begin{aligned}
& \left(1-p\left(e_{0}\right)\right) u\left(w-\beta_{0}\right)+p\left(e_{0}\right) u\left(w-d+\alpha_{0}\right)-e_{0} \\
& \quad \leqslant u(w+(w+v) / q)-e_{0} \\
& \quad<u(w-d)-\underline{e} \\
& \quad \leqslant(1-\bar{p}) u(w)+\bar{p} u(w-d)-\underline{e} ;
\end{aligned}
$$

Therefore $\left(e_{0}, \alpha_{0}, \beta_{0}\right)$ is strictly dominated by $(\underline{e}, 0,0)$ and hence by any element of $\bar{\lambda}(v, q)$. The desired result follows immediately.

Q.E.D.

Proof of Lemma 2.1: Consider the set $V=\{v \mid(1-p(e)) \beta-p(e) \alpha \geqslant 0$ for some $(e, \alpha, \beta) \in \lambda(v, \bar{q})\}$. Since $\bar{q} \geqslant p(e) /(1-p(e))$ for all $e, \quad(e, \alpha, \beta) \in \lambda(0, \bar{q})$ implies $(1-p(e)) \beta-p(e) \alpha \geqslant 0$. Hence Lemma A.2 implies $0 \in V$. Further, $v \geqslant \bar{q} d$ and $(e, \alpha, \beta) \in \lambda(v, \bar{q})$ imply $\beta=0, \alpha=v / \bar{q} \geqslant d$, trivially, and therefore $e=e$, by the same argument as in Lemma A.l. Thus $v \geqslant \bar{q} d$ and $(e, \alpha, \beta) \in \bar{\lambda}(v, \bar{q})$ imply $(1-p(e)) \beta-p(e) \alpha=-\bar{p} v / \bar{q}<0$, hence $v \notin V$. Thus $V$ is nonempty and bounded above. Let $v_{0}$ be the least upper bound of $V$. By Lemma A.2, there exists $\left(e_{0}, \alpha_{0}, \beta_{0}\right) \in \lambda\left(v_{0}, \bar{q}\right)$ such that $\left(1-p\left(e_{0}\right)\right) \beta_{0}-p\left(e_{0}\right) \alpha_{0} \geqslant 0$. Since expected profits are nonzero, by assumption, this implies $\left(1-p\left(e_{0}\right)\right) \beta_{0}-p\left(e_{0}\right) \alpha_{0}>0$. For $v \in\left(v_{0}, \bar{q} d\right),(e, \alpha, \beta) \in \lambda(v, \bar{q})$ implies $(1-p(e)) \beta-p(e) \alpha<0$, hence $(1-p(e)) \bar{q}-p(e)<0$, and Lemma A.l implies $e=\underline{e}, \beta=(\underline{q} d-v) /(1+\bar{q}), \alpha=(d-v) /(1+q)$.

Q.E.D. 
Proof of Proposition 5.a: Zero insurance with the effort level $\overline{\boldsymbol{e}}$ yields the utility (A.5a) $-(1-p) \exp (-w)-\underline{p} \exp (-w+d)-\bar{e}$.

Full insurance at the rate $\bar{q}$, together with the effort level $\underline{e}$ yields the utility:

$$
-\exp \left(-w+\frac{\bar{q} d}{1+\bar{q}}\right)-\underline{e} \text {. }
$$

Observe that $\bar{q} /(1+\bar{q})=\bar{p}$. Therefore the difference between (A.5a) and (A.5b) is strictly positive if :

$$
(1-\underline{p})(\exp (d)-1)-\exp (d)[1-\exp (-(1-\bar{p}) d)]>(\bar{e}-\underline{e}) \exp (w) .
$$

By assumption, $(1-\underline{p})(\exp (d)-1)>(\bar{e}-\underline{e}) \exp (w)$. Therefore there exists $\varepsilon_{1}>0$, such that for $\bar{p}>1-\varepsilon_{1},(\mathrm{~A} .5 \mathrm{a})$ is strictly greater than (A.5b).

Next, I shall discuss some properties of the demand relation $\lambda$. Given $v, q$, and the effort level $\bar{e}$, the optimal insurance terms are easily calculated as :

$$
\alpha=\frac{1}{1+q}\left[d+v-\ln \frac{q}{q}\right], \beta=\frac{q}{1+q}\left[d-\ln \frac{q}{q}\right]-\frac{1}{1+q} v,
$$

yielding the expected utility :

$$
-\exp \left[-w+\frac{q d-v}{1+q}\right]\left[(1-\underline{p})\left(\frac{q}{q}\right)^{-\frac{q}{1+q}}+\underline{p}\left(\frac{q}{q}\right)^{\frac{1}{1+q}}\right]-\bar{e} .
$$

Similarly, for $v, q$, and the effort level $\underline{e}$, the optimal insurance terms are:

$$
\alpha=\frac{1}{1+q}\left[d+v-\ln \frac{q}{q}\right], \beta=\frac{q}{1+q}\left[d-\ln \frac{q}{q}\right]-\frac{1}{1+q} v,
$$

yielding the expected utility :

$$
-\exp \left[-w+\frac{q d-v}{1+q}\right]\left[(1-\bar{p})\left(\frac{q}{\bar{q}}\right)^{-\frac{q}{1+q}}+\bar{p}\left(\frac{q}{\bar{q}}\right)^{\frac{1}{1+q}}\right]-\bar{e} .
$$

Lemma A.3: Under the assumptions of Proposition 5, assume that for some $v^{*} \geqslant 0, q^{*} \leqslant \bar{q}$, there exist $\left(\alpha_{1}^{*}, \beta_{1}^{*}\right),\left(\alpha_{2}^{*}, \beta_{2}^{*}\right)$, such that $\left(\underline{e}, \alpha_{1}^{*}, \beta_{1}^{*}\right) \in \lambda\left(v^{*}, q^{*}\right)$ and $\left(\bar{e}, \alpha_{2}^{*}, \beta_{2}^{*}\right) \in \lambda\left(v^{*}, q^{*}\right)$. Then $v>v^{*}$ and $(e, \alpha, \beta) \in \lambda\left(v, q^{*}\right)$ imply $e=\underline{e}, \alpha+\beta=d+\ln \left(\bar{q} / q^{*}\right) \geqslant d$.

Proof: The lemma follows from Lemma A.1 and the observation that at the point $\left(v^{*}, q^{*}\right)$ at which (A.6a) and (A.6b) are equal, the difference between (A.6a) and (A.6b) is strictly decreasing in $v$.

Q.E.D.

Proof of Proposition 5.b: Let $\bar{p}>1-\varepsilon_{1}$, where $\varepsilon_{1}>0$ is given in the proof of Proposition 5.a. By Proposition 5.a, we have $\left(\underline{e}, \frac{d}{1+\bar{q}}, \frac{\bar{q} d}{1+\bar{q}}\right) \notin \lambda(0, \bar{q})$, so that Lemma A.1 implies $(e, \alpha, \beta) \in \lambda(0, \bar{q})$ only if $\mathrm{e}=\bar{e}$. On the other hand, Lemma A.l also implies $(e, \alpha, \beta) \in \lambda(0, q)$ only if $\alpha+\beta>d$ and $e=e$. By Lemma A.2, there exist $q^{*} \in(q, \bar{q})$ and $\left(\alpha_{1}^{*}, \beta_{1}^{*}\right),\left(\alpha_{2}^{*}, \beta_{2}^{*}\right)$, such that $\left(\underline{e}, \alpha_{1}^{*}, \beta_{1}^{*}\right) \in \lambda\left(0, q^{*}\right)$ and $\left(\bar{e}, \alpha_{2}^{*}, \beta_{2}^{*}\right) \in \lambda\left(0, q^{*}\right)$. By Lemma A.3, it follows that for $v>0,(e, \alpha, \alpha) \in \lambda\left(v, q^{*}\right)$ implies $e=e$ and $\alpha+\beta>d$. Thus, we have the constellation of Figure 10a in Professor Stiglitz's lecture, and the result follows from his argument.

Q.E.D. 
I shall now study the curve $\mathrm{AA}^{\prime}$ of Figure 6 , i.e. the locus of points at which one is indifferent between staying put and moving up to full insurance at the rate $\bar{q}$. Let $\left(\alpha_{0}, \beta_{0}\right)$ be defined as in the proof of Proposition 4. and let $v_{0}=\bar{q} \alpha_{0}-\beta_{0}$. Then one has:

Lemma $A .4$ : Let $\bar{p}>1-\varepsilon_{1}$. For $v \in\left[0, v_{0}\right]$, there exists a unique pair $(\alpha(v), \beta(v))$, such that $\beta(v)=\bar{q} \alpha(v)-v$ and

$$
\begin{aligned}
& -(1-\underline{p}) \exp (-w+\beta(v))-\underline{p} \exp (-w+d-\alpha(v))-\bar{e} \\
& =-\exp \left(-w+\frac{\bar{q}}{1+\bar{q}} d-\frac{\bar{q}}{1+\bar{q}} \alpha(v)+\frac{1}{1+\bar{q}} \beta(v)\right)-\underline{e},
\end{aligned}
$$

whereas $(\alpha, \beta)>(\alpha(v), \beta(v))$ implies :

$$
\begin{aligned}
& -(1-\underline{p}) \exp (-w+\beta)-\underline{p} \exp (-w+d-\alpha)-\bar{e} \\
& <-\exp \left(-w+\frac{\bar{q}}{1+\bar{q}} d-\frac{1}{1+\bar{q}} \beta\right)-\underline{e} .
\end{aligned}
$$

The map $v \rightarrow(\alpha(v), \beta(v))$ is continuous. Moreover, $\alpha\left(v_{0}\right)=\alpha_{0}$ and $\beta\left(v_{0}\right)=\beta_{0}$.

Remark: The left hand side of (A.7a) gives the expected utility of the triple $(\bar{e}, \alpha(v), \beta(v))$; the right hand side the expected utility of moving from $(\alpha(v), \beta(v))$ to full insurance at the rate $\bar{q}$.

Proof: For $\bar{p}>1-\varepsilon_{1}$, we have $\left(\underline{e}, \frac{d}{1+\bar{q}}, \frac{\bar{q} d}{1+\bar{q}}\right) \notin \lambda(0, \bar{q})$, hence $v_{0}>0$. For $v \leqslant v_{0}$, there exist $\underline{\alpha}(v), \underline{\beta}(v)$, such that $(\bar{e}, \underline{\alpha}(v), \underline{\beta}(v)) \in \lambda(v, \bar{q})$, by definition of $v_{0}$ and by Lemma A.3. For $v \leqslant v_{0}$ and any $\alpha$, set $\beta=\bar{q} \alpha-v$ and define :

$$
\begin{aligned}
f(v, \alpha)= & -(1-\underline{p}) \exp (-w+\bar{q} \alpha-v)-\underline{p} \exp (-w+d-\alpha)-\bar{e} \\
& +\exp \left(-w+\frac{\bar{q} d-v}{1+\bar{q}}\right)+\underline{e} .
\end{aligned}
$$

For $v \leqslant v_{0}$, one has $f(v, \underline{\alpha}(v)) \geqslant 0$, and $f\left(v, \frac{d+v}{1+\bar{q}}\right)<0$. Moreover, $f(v, \alpha)$ is continuous in $v$ and $\alpha$ as well as strictly decreasing in $\alpha$ on the set $\{(v, \alpha) \mid \alpha \geqslant \underline{\alpha}(v)\}$. By the intermediate value theorem and strict monotonicity, there exists a unique $\alpha(\bar{v})$ such that $f(v, \alpha(v))=0$. The pair $(\alpha(v), \bar{q} \alpha(v)-v)$ clearly satisfies the conditions of the lemma. Continuity of the map $v \rightarrow(\alpha(v), \bar{q} \alpha(v)-v)$ follows from the continuity of $f$. Finally, the definition of $v_{0}$ implies $\alpha_{0}=\alpha\left(v_{0}\right), \beta_{0}=\beta\left(v_{0}\right)$.

Q.E.D.

Lemma A.4 defines the curve $\mathrm{AA}^{\prime}$ of Figure 6 parametrically in terms of the income level $v$. The following lemma shows that for $\bar{p}$ sufficiently close to 1 , the curve $\mathrm{AA}^{\prime}$ is given by a function $\alpha \rightarrow \beta^{*}(\alpha)$ whose slope is negative with an absolute value greater than 1, i.e. the curve $\mathrm{AA}^{\prime}$ is at least as steep as the negative $45^{\circ}$ - line.

Lemma A.5: Under the assumptions of Proposition 5, there exists $\varepsilon_{2} \in\left(0, \varepsilon_{1}\right)$, such that for $\bar{p}>1-\varepsilon_{2}$, the function $v \rightarrow \alpha(v)$ defined by (A.7a) is strictly increasing, and the implicit function $\alpha \rightarrow \beta^{*}(\alpha)=\beta\left(\alpha^{-1}(\alpha)\right)$ has a slope $\frac{d \beta^{*}}{d \alpha}<-\left(1+\varepsilon_{2}\right)$.

Remark: Lemma A.5 implies in particular that $\beta^{*}(\alpha)>q \alpha$ for all $\alpha<\alpha_{0}$, so that for all $v<v_{0}$ the pair $(\alpha(v), \beta(v))$ lies in the region of positive profits. 
Proof: Let $\eta_{1}=\min \left(\varepsilon_{1},(1-\underline{p}) \exp (-d)\right)>0$, so that $\bar{p}>1-\eta_{1}$ implies $\bar{p}>1-\varepsilon_{1}$ and $(1-\underline{p})>(1-\bar{p}) \exp \left(\frac{\bar{q} \delta}{1+\bar{q}}\right)$ for all $\delta \leqslant d$. For $\bar{p}>1-\eta_{1}$, the function $f$ in the proof of Lemma A.4 is strictly increasing in $v$. Since $f$ is strictly decreasing in $\alpha$ for $\alpha \geqslant \alpha(v)$, the function $v \rightarrow \alpha(v)$ satisfying $f(v, \alpha(v))=0$ for all $v$ is strictly increasing. Then the function $\beta^{*}=\beta \circ \alpha^{-1}$ is well defined. Applying the implicit function theorem to (A.7a), we have :

$$
\frac{d \beta^{*}}{d \alpha}=-\frac{\bar{p} \exp \left(\frac{\bar{q}}{1+\bar{q}} \delta\right)-\underline{p} \exp (\delta)}{(1-\underline{p})-(1-\bar{p}) \exp \left(\frac{\bar{q}}{1+\bar{q}} \delta\right)},
$$

where $\delta=d-\alpha-\beta \varepsilon(0, d)$. Thus,

$$
\begin{aligned}
\frac{d \beta^{*}}{d \alpha} & =-1-\frac{-(1-\underline{p})-\underline{p} \exp (\delta)+\exp \left(\frac{\bar{q}}{1+\bar{q}} \delta\right)}{(1-\underline{p})-(1-\bar{p}) \exp \left(\frac{\bar{q}}{1+\bar{q}} \delta\right)} \\
& =-1-\frac{(\bar{e}-\underline{e}) \exp (w-\beta)}{(1-\underline{p})-(1-\bar{p}) \exp \left(\frac{\bar{q}}{1+\bar{q}} \delta\right)}<-1-\frac{\bar{e}-\underline{e}}{1-\underline{p}},
\end{aligned}
$$
by substitution from (A.7a). Set $\varepsilon_{2}=\min \left(\eta_{1}, \frac{\bar{e}-\underline{e}}{1-\underline{p}}\right)$, and the lemma follows
immediately.

Q.E.D.

I shall now specify the insurance terms $\left(\alpha_{n}, \beta_{n}\right)$ for Proposition 5c. Recall that $\hat{\lambda}(\underline{\alpha}, \underline{\beta})$ is the set of triples $(\hat{e}, \hat{\alpha}, \hat{\beta})$ that maximize expected utility along the ray $\{(\alpha, \beta) \mid(\alpha, \beta)=(\underline{\alpha}, \underline{\beta})+\delta(1, \bar{q}), \delta \geqslant 0\}$.

Lemma A.6: Under the assumptions of Proposition $5, \bar{p}>1-\varepsilon_{2}$ implies the following. For every integer $n$, there exist insurance terms $\left(\alpha_{n}, \beta_{n}\right)$, such that : (i) $\beta_{n}=\beta^{*}\left(\alpha_{n}\right)$; (ii) the consumer is indifferent between the triples $\left(\vec{e}, \alpha_{n}, \beta_{n}\right)$ and $\left(\bar{e}, \frac{n-1}{n} \alpha_{n}, \frac{n-1}{n} \beta_{n}\right)$, i.e.

$$
\begin{aligned}
& -(1-\underline{p}) \exp \left(-w+\beta_{n}\right)-\underline{p} \exp \left(-w+d-\alpha_{n}\right) \\
& =-(1-\underline{p}) \exp \left(-w+\frac{n-1}{n} \beta_{n}\right)-\underline{p} \exp \left(-w+d-\frac{n-1}{n} \alpha_{n}\right)
\end{aligned}
$$

(iii) $\hat{\lambda}\left(\frac{n-1}{n} \alpha_{n}, \frac{n-1}{n} \beta_{n}\right)=\left\{\left(\bar{e}, \frac{n-1}{n} \alpha_{n}, \frac{n-1}{n} \beta_{n}\right)\right\}$.

Proof: Recall that for $\bar{p}>1-\varepsilon_{2}$, the function $v \rightarrow \alpha(v)$ is strictly increasing, by Lemma A.5. For $\alpha \in[\alpha(v), \alpha(v)]$ define :

$$
\begin{aligned}
\hat{f_{n}}(\alpha)= & -(1-\underline{p}) \exp \left(-w+\beta^{*}(\alpha)\right)-\underline{p} \exp (-w+d-\alpha) \\
& +(1-\underline{p}) \exp \left(-w+\frac{n-1}{n} \beta^{*}(\alpha)\right)+\underline{p} \exp \left(-w+d-\frac{n-1}{n} \alpha\right) .
\end{aligned}
$$


By definition of $e_{2}, \bar{p}>1-\varepsilon_{2}$ implies further that the slope of the consumer's indifference curves in the high effort region, $\frac{d \beta}{d \alpha}=\frac{p}{1-\underline{p}} e^{d-\alpha-\beta}$, is strictly less than the premium-benefit ratio $\bar{q}=\frac{\bar{p}}{1-\bar{p}}$; in the high effort region, one must therefore have $\hat{\lambda}(\alpha, \beta)=\{(\bar{e}, \alpha, \beta)\}$, i.e. the consumer buys no additional insurance at the premium-benefit ratio $\bar{q}$. In particular, $\hat{\lambda}(0,0)=\{(\bar{e}, 0,0)\}$, hence $0 \geqslant \underline{\alpha}\left(v_{0}\right)$ and $f_{n}(\alpha(0))<0$. Moreover $\beta\left(v_{0}\right)-\beta^{*}\left(\alpha\left(v_{0}\right)\right)=\underline{q} \alpha\left(v_{0}\right) \frac{p}{1-p} \alpha\left(v_{0}\right)$, by definition of $v_{0}$, and therefore $f_{n}\left(\alpha\left(v_{0}\right)\right)>0$. Clearly $f_{n}$ is continuous on the interval $\left[\alpha(0), \alpha\left(v_{0}\right)\right]$. By the intermediate value theorem, there exists $\alpha_{n} \in\left(\alpha(0), \alpha\left(v_{0}\right)\right)$, such that $f_{n}\left(\alpha_{n}\right)=0$. Set $\beta_{n}=\beta^{*}\left(\alpha_{n}\right)$ to obtain (i) and (ii). (iii) then follows because $\left(\frac{n-1}{n} \alpha_{n}, \frac{n-1}{n} \beta_{n}\right)<\left(\alpha_{n}, \beta_{n}\right)$ lies in the high effort region in which the consumer's indifference curves have a slope less than $\bar{q}$.

Q.E.D.

Proof of Proposition 5.c: Let $\bar{p}>1-\varepsilon_{2}$ and consider the strategy array $G$ that is given by (8a) $-(8 \mathrm{c})$, with $\left(\alpha_{n}, \beta_{n}\right)$ given by Lemma A.6. Define $\bar{c}=\left\{\bar{\alpha}_{i}, \bar{\beta}_{i}\right\}_{i=1}^{\infty}$, such that $\left(\bar{\alpha}_{i}, \bar{\beta}_{i}\right)=\frac{1}{n}\left(\alpha_{n}, \beta_{n}\right)$ for $i=1,2, \ldots n,\left(\bar{\alpha}_{i}, \bar{\beta}_{i}\right)=(0,0)$ for $i \geqslant n+1$. By Lemma A.6 (i), $(\bar{e}, \bar{c}) \in \emptyset(G)$, with $\bar{\pi}_{i}=\frac{1}{n}\left[(1-\underline{p}) \beta_{n}-\underline{p} \alpha_{n}\right]>0$ for $i=1,2, \ldots n, \bar{\pi}_{i}=0$ for $i \geqslant n+1$.

By contradiction, suppose that $(G, \bar{\pi})$ is not an MCE. Then there exists a firm $j$, a strategy $h$, a consumer choice $(\hat{e}, \hat{c})=\left(\hat{e},\left\{\hat{\alpha}_{i}, \hat{\beta}_{i}\right\}_{i=1}^{\infty}\right) \in \emptyset\left(H_{j}(h, G)\right)$, such that $\hat{\pi}_{j}=\bar{\pi}_{j}(\hat{e}, \hat{c})>\bar{\pi}_{j}$, hence $\left(\hat{\alpha}_{j}, \hat{\beta}_{j}\right) \neq 0$. Since firms $i \geqslant n+1, i \neq j$, offer arbitrary amounts of insurance at the premium-benefit ratio $\bar{q},\left(\hat{\alpha}_{j}, \hat{\beta}_{j}\right) \neq 0$ implies $\hat{\beta}_{j} / \hat{\alpha}_{j} \leqslant \bar{q}$. If $\beta_{j} / \hat{\alpha}_{j}=\bar{q}$, we have $\hat{e}=e$ because $\bar{p}>1-\varepsilon_{2}$ implies that in the high effort region no contract with a premium-benefit ratio $\bar{q}$ is purchased (see proof on Lemma A.6). Then we would have $\hat{\pi}_{j}=0$, contrary to the assumption that $\hat{\pi}_{j}>\bar{\pi}_{j} \geqslant 0$. Therefore we may assume $\hat{\beta}_{j} / \hat{\alpha}_{j}<\bar{q}, \hat{e}=\bar{e}$, and hence :

$\sum_{i} \hat{\alpha}_{i} \leqslant \alpha\left(\bar{q} \sum_{i} \hat{\alpha}_{i}-\sum_{i} \hat{\beta}_{i}\right), \sum_{i} \hat{\beta}_{i} \leqslant \beta\left(\bar{q} \sum_{i} \hat{\alpha}_{i}-\sum_{i} \hat{\beta}_{i}\right)$, by Lemma A.4.

Assume first that $j \geqslant n+1$ and define $\bar{\alpha}=\sum_{i=n+1}^{\infty} \hat{\alpha}_{i}, \bar{\beta}=\sum_{i=n+1}^{\infty} \hat{\beta}_{i}$. Let $\sum_{i=1}^{n}\left(\hat{\alpha}_{i}, \hat{\beta}_{i}\right)=\frac{m}{n}\left(\alpha_{n}, \beta_{n}\right)$ be the aggregate insurance purchase from firms $1,2, \ldots n$. For $m=n$, Lemma A.6(i) implies $\sum_{i=1}^{n} \hat{\alpha}_{i}>\alpha\left(\bar{q} \sum_{i} \hat{\alpha}_{i}-\sum_{i} \hat{\beta}_{i}\right), \sum_{i=1}^{n} \hat{\beta}_{i}>\beta\left(\bar{q} \sum_{i} \hat{\alpha}_{i}-\sum_{i} \hat{\beta}_{i}\right)$, contrary to our preceding conclusion. Therefore we have $m \leqslant n-1$.

The chosen triple $\left(\bar{e}, \frac{m}{n} \alpha_{n}+\bar{\alpha}, \frac{m}{n} \beta_{n}+\bar{\beta}\right)$ must be weakly preferred to the triple $\left(\bar{e}, \alpha_{n}, \beta_{n}\right)$, which is still available. Therefore there exists $\delta \geqslant 0$, such that the consumer is indifferent between $\left(\bar{e}, \frac{m}{n} \alpha_{n}+\bar{\alpha}-\delta, \frac{m}{n} \beta_{n}+\bar{\beta}+\delta\right)$ and $\left(\bar{e}, \alpha_{n}, \beta_{n}\right)$. The assumption of constant absolute risk aversion implies that he is then also indifferent between the triples $\left(\bar{e}, \alpha_{n}+\delta, \beta_{n}-\delta\right)$ and $\left(\bar{e}, \frac{m}{n} \alpha_{n}+\bar{\alpha}, \frac{m}{n} \beta_{n}+\bar{\beta}\right)$. By Lemmas A.5 and A.6(i), the triple 
$\left(\bar{e}, \alpha_{n}+\delta, \mathrm{b}_{n}-\delta\right)$ and therefore the triple $\left(\bar{e}, \frac{m}{n} \alpha_{n}+\bar{\alpha}, \frac{m}{n} \beta_{n}+\bar{\beta}\right)$ are weakly dominated by the triple $\left(\underline{e}, \frac{d+\hat{v}}{1+\bar{q}}, \frac{\bar{q} d-\hat{v}}{1+\bar{q}}\right), \hat{v}=\bar{q} \alpha_{n}-\beta_{n}+\left(1+\frac{n}{q}\right) \delta$, that is attained by supplementing the pair $\left(\alpha_{n}+\delta, \beta_{n}-\delta\right)$ at the premium-benefit ratio $\bar{q}$ up to full insurance. I will show that the triple $\left(\underline{e}, \frac{d+\hat{v}}{1+\bar{q}}, \frac{\bar{q} d-\hat{v}}{1+\bar{q}}\right)$ in turn is strictly dominated by the triple $\left(e, \frac{d+\bar{v}}{1+\bar{q}}, \frac{\bar{q} d-\bar{v}}{1+\bar{q}}\right), \bar{v}=\bar{q}\left(\alpha_{n}+\bar{\alpha}\right)-\left(\beta_{n}+\bar{\beta}\right)$, that is attained by supplementing the available combination $\left(\alpha_{n}+\bar{\alpha}, \beta_{n}+\bar{\beta}\right)$ at the premium-benefit ratio $\bar{q}$ up to full insurance. It will then follow that $\left(\bar{e}, \frac{m}{n} \alpha_{n}+\bar{\alpha}, \frac{m}{n} \beta_{n}+\bar{\beta}\right)$ is strictly dominated by $\left(\underline{e}, \frac{d+\bar{v}}{1+\bar{q}}, \frac{\bar{q} d-\bar{v}}{1+\bar{q}}\right)$, contrary to the assumption that $\left(\bar{e}, \frac{m}{n} \alpha_{n}+\bar{\alpha}, \frac{m}{n} \beta_{n}+\bar{\beta}\right)$ is the optimal choice for the consumer.

The triple $\left(\underline{e}, \frac{d+\bar{v}}{1+\bar{q}}, \frac{\bar{q} d-\bar{v}}{1+\bar{q}}\right)$ dominates $\left(\underline{e}, \frac{d+\hat{v}}{1+\bar{q}}, \frac{\bar{q} d-\hat{v}}{1+\bar{q}}\right)$, if and only if $\bar{v}>\hat{v}$, or $\bar{\beta}+\delta<\bar{q}(\bar{\alpha}-\delta)$. To obtain this inequality, recall that $\left(\bar{e}, \frac{m}{n} \alpha_{n}+\bar{\alpha}-\delta, \frac{m}{n} \beta_{n}+\bar{\beta}+\delta\right)$ yields the same utility as $\left(\bar{e}, \alpha_{n}, \beta_{n}\right)$. By Lemma A.6(ii) and strict concavity of the utility function $u$, the triple $\left(\bar{e}, \alpha_{n}, \beta_{n}\right)$ and therefore also $\left(\bar{e}, \frac{m}{n} \alpha_{n}+\bar{\alpha}-\delta, \frac{m}{n} \beta_{n}+\bar{\beta}+\delta\right)$ is weakly preferred to the triple $\left(\bar{e}, \frac{m}{n} \alpha_{n}, \frac{m}{n} \beta_{n}\right)$. Since indifference curves in the high effort region have a slope less than $\bar{q}$, we must have $\bar{\beta}+\delta<\bar{q}(\bar{\alpha}-\delta)$, hence $\bar{v}>\hat{v}$, as was to be shown.

It remains to examine the case $j \leqslant n$. From Lemma A.6(i) - (iii) one easily finds that the insurance terms $\frac{1}{n}\left(\alpha_{n}, \beta_{n}\right)$ maximize firm $j$ 's profits $(1-\underline{p}) \beta-\underline{p} \alpha$ over the set of terms $(\alpha, \beta)$, such that (a) $\frac{n-1}{n} \beta_{n}+\beta \leqslant \beta^{*}\left(\frac{n-1}{n} \alpha_{n}+\alpha\right)$, and (b) the consumer purchases the contract $\left((\alpha, \beta), s_{j}\right)$ only if his overall purchases make him at least as well off as the insurance terms $\left(\frac{n-1}{n} \alpha_{n}, \frac{n-1}{n} \beta_{n}\right)$ that he can have from firms $i=1,2, \ldots n, i \neq j$. Since $\left(\hat{\alpha}_{j}, \hat{\beta}_{j}\right)$ yields higher profits than $\frac{1}{n}\left(\alpha_{n}, \beta_{n}\right)$, we must have $\frac{n-1}{n} \beta_{n}+\hat{\beta}_{n}>\beta^{*}\left(\frac{n-1}{n} \alpha_{n}+\hat{\alpha}_{j}\right)$. But then the argument for the case $j \geqslant n+1$ can be repeated to show that the consumer wishes to buy from all the firms $i=1,2, \ldots n$ and then to supplement at the premium-benefit ratio $\bar{q}$ up to full insurance. His effort level then is $\underline{e}$, and firm $j$ 's profits $\hat{\pi}_{j}$ are negative, in contradiction to the assumption that $\hat{\pi}_{j}>\bar{\pi}_{j}$. Both, the assumption that $j \geqslant n+1$ and the assumption that $j \leqslant n$, are therefore false, and the proof of Proposition 5.c is complete.

Q.E.D.

Proof of Proposition 5.d: Let $\left(\alpha_{\infty}, \beta_{\infty}\right)$ be a limit point of the sequence $\left\{\alpha_{n}, \beta_{n}\right\}_{n=1}^{\infty}$ of Lemma A.6. Then $\left(\alpha_{\infty}, \beta_{\infty}\right)$ is also a limit point of the sequence $\left\{\frac{n-1}{n} \alpha_{n}, \frac{n-1}{n} \beta_{n}\right\}_{n=1}^{\infty}$. For each $n$, the consumer is indifferent between $\left(\bar{e}, \alpha_{n}, \beta_{n}\right)$ and $\left(\bar{e}, \frac{n-1}{n} \alpha_{n}, \frac{n-1}{n} \beta_{n}\right)$. By concavity 
of the utility function $u$, the slope of his indifference curve at $\left(\bar{e}, \frac{n-1}{n} \alpha_{n}, \frac{n-1}{n} \beta_{n}\right)$ exceeds $\beta_{n} / \alpha_{n}$, whereas the slope of his indifference curve at $\left(\bar{e}, \alpha_{n}, \beta_{n}\right)$ is less than $\beta_{n} / \alpha_{n}$. In the limit, the slope of his indifference curve at $\left(\alpha_{\infty}, \beta_{\infty}\right)$ must therefore be equal to $\beta_{\infty} / \alpha_{\infty}$. On the other hand, the slope of his indifference curve at $\left(\alpha_{0}, \beta_{0}\right)$ is strictly greater than $\underline{q}=\beta_{0} / \alpha_{0}$. Therefore, $\left(\alpha_{0}, \beta_{0}\right)$ cannot be a limit point of the sequence $\left\{\alpha_{n}, \beta_{n}\right\}_{n=1}^{\infty}$.

Q.E.D.

Proof of Proposition 5.e : From the proof of Proposition 5.b, recall that $\left(\bar{e}, \alpha_{2}^{*}, \beta_{2}^{*}\right) \in \lambda\left(0, q^{*}\right)$ is the equilibrium allocation in the positive profit price equilibrium. By contradiction, suppose that for infinitely many $n$ the allocation $\left(\bar{e}, \alpha_{2}^{*}, \beta_{2}^{*}\right)$ is weakly preferred to the allocation $\left(\bar{e}, \alpha_{n}, \beta_{n}\right)$. Observe that $\alpha_{n}+\beta_{n} \leqslant d$ for all $n$, and, taking a subsequence if necessary, let $\left(\alpha_{n}, \beta_{n}\right) \rightarrow\left(\alpha_{\infty}, \beta_{\infty}\right)$. Then $\left(\bar{e}, \alpha_{2}^{*}, \beta_{2}^{*}\right)$ is weakly preferred to $\left(\bar{e}, \alpha_{\infty}, \beta_{\infty}\right)$. From the proof of Proposition 5.d, we know that the slope of the consumer's indifference curve at $\left(\alpha_{\infty}, \beta_{\infty}\right)$ is $\beta_{\infty} / \alpha_{\infty}$. Therefore, $\left(\bar{e}, \alpha_{\infty}, \beta_{\infty}\right)$ dominates all triples $(e, \alpha, \beta)$ with $\alpha<\alpha_{\infty}$ and $\beta / \alpha \leqslant \beta_{\infty} / \alpha_{\infty}$. Since $\left(\bar{e}, \alpha_{2}^{*}, \beta_{2}^{*}\right)$ dominates $\left(\bar{e}, \alpha_{\infty}, \beta_{\infty}\right)$, it follows that $q^{*} \leqslant \beta_{\infty} / \alpha_{\infty}$.

For each $n$, one easily verifies that the function $f_{n}$ in the proof of Lemma A. 6 satisfies $\hat{f_{n}}\left(\alpha_{\infty}\right)>0$. Repeating the intermediate value argument in the proof of Lemma A.6, we may therefore find $\left(\bar{\alpha}_{n}, \bar{\beta}_{n}\right)$ satisfying Lemma A.6(i) - (iii) such that $\bar{\alpha}_{n} \leq \alpha_{\infty}$ and $\bar{\beta}_{n}>\beta_{\infty}$. Repeating the proof of Proposition 5.c, we find that $(8 \mathrm{a})-(8 \mathrm{c})$ with $\left(\bar{\alpha}_{n}, \bar{\beta}_{n}\right)$ in the place of $\left(\alpha_{n}, \beta_{n}\right)$ again yields an MCE without communication.

To obtain a contradiction, suppose now that firm $n+1$ replaces the inactive strategy $g_{n+1}$ of $(8 \mathrm{~b})$ with the new strategy $h$, such that $h(\gamma)=\left(\left(\alpha_{2}^{*}, \beta_{2}^{*}\right), 0\right)$ for all $\gamma$. Since $\left(\bar{e}, \alpha_{2}^{*}, \beta_{2}^{*}\right) \in \lambda\left(0, q^{*}\right)$ and $\beta_{2}^{*} / \alpha_{2}^{*}=q^{*}$ is strictly less than the premium-benefit ratio $\bar{\beta}_{n} / \bar{\alpha}_{n}$ of firms $1,2, \ldots n$ and the premium-benefit ratio $\bar{q}$ of firms $n+2, n+3, \ldots$, the consumer will simply buy the contract $\left(\left(\alpha_{2}^{*}, \beta_{2}^{*}\right), 0\right)$ from firm $n+1$, choose the high level of effort, and buy no other contract. Firm $n+1$ now makes strictly positive profits. Thus the strategy $h$ dominates the original strategy $g_{n+1}$, contrary to the previous statement that $(8 \mathrm{a})-(8 \mathrm{c})$, with $\left(\bar{\alpha}_{n}, \bar{\beta}_{n}\right)$ in the place of $\left(\alpha_{n}, \beta_{n}\right)$, yields an MCE. The assumption that $\left(\bar{e}, \alpha_{2}^{*}, \beta_{2}^{*}\right)$ dominates $\left(\bar{e}, \alpha_{n}, \beta_{n}\right)$ for infinitely many $n$ has thus led to a contradiction and must be false.

Q.E.D. 


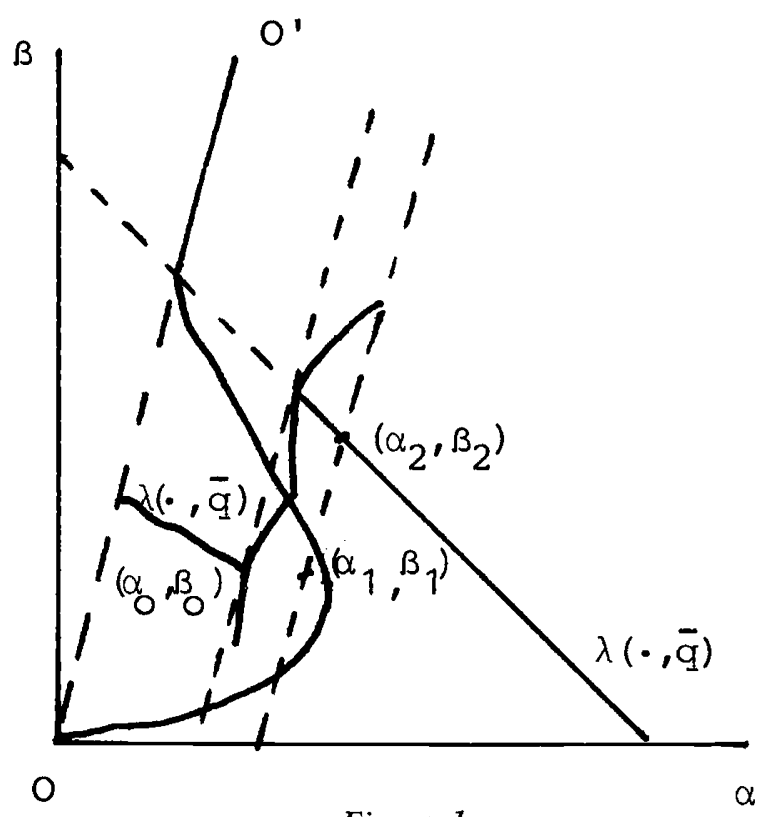

Figure 1

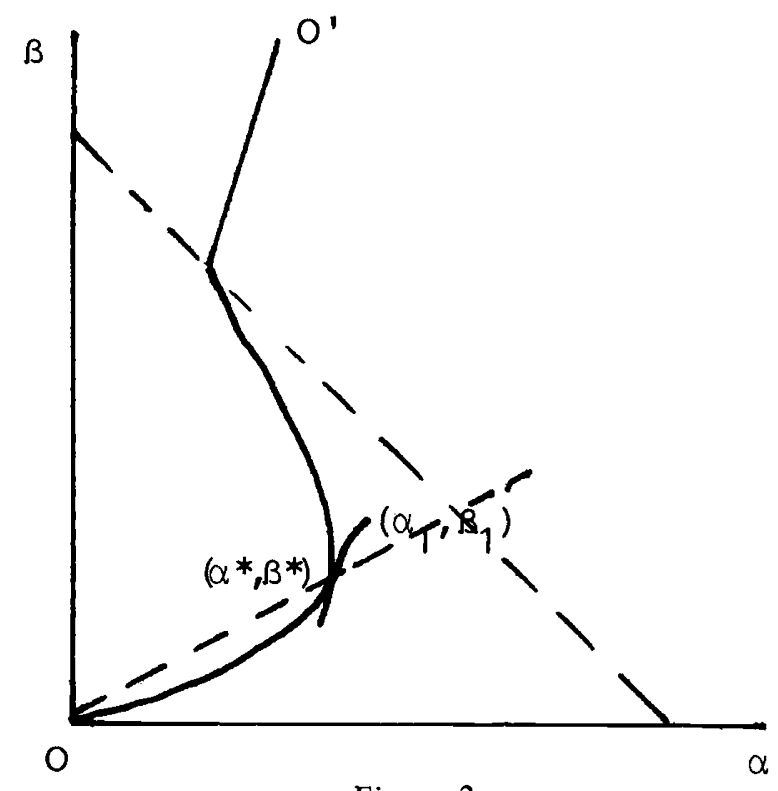

Figure 2 


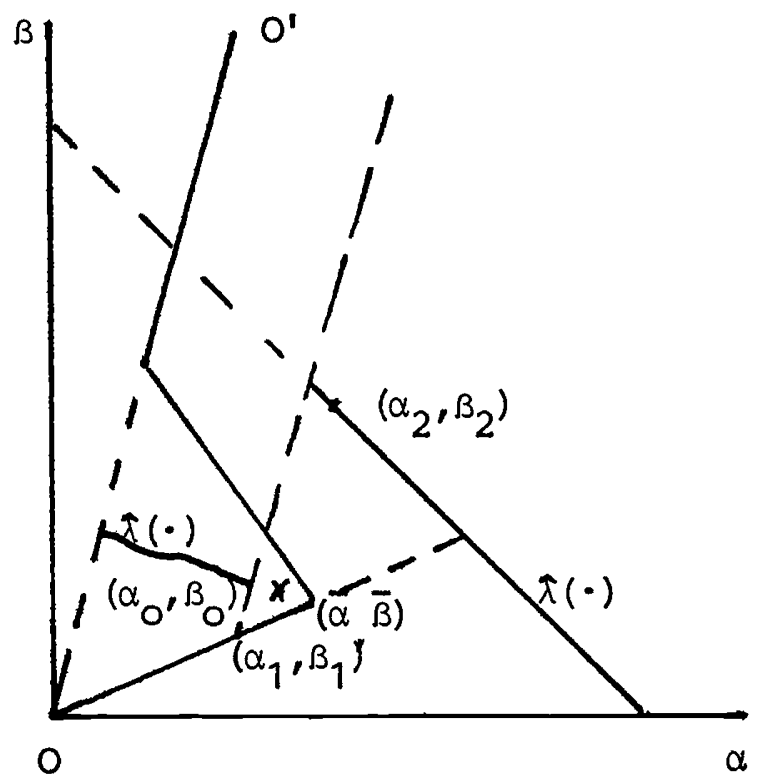

Figure 3

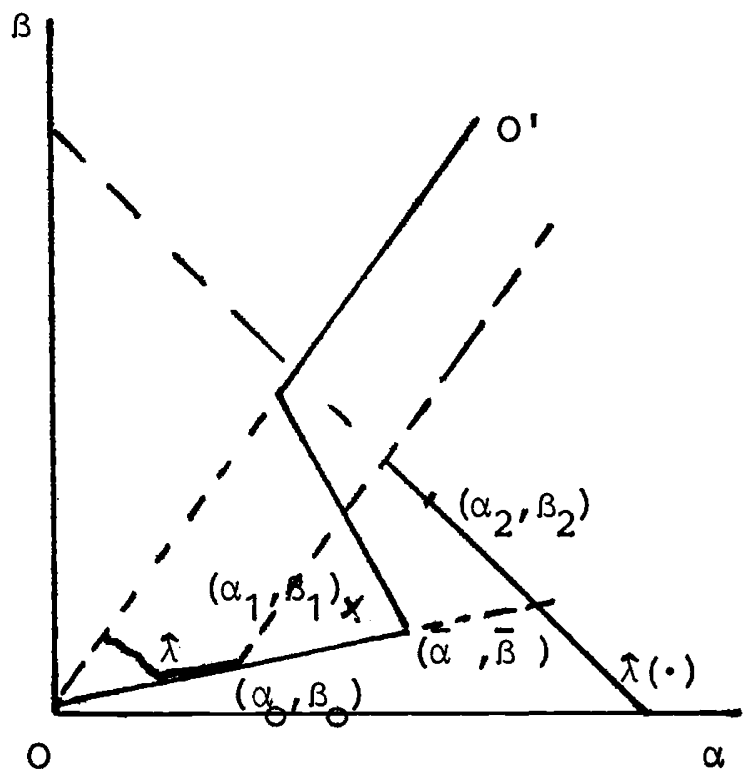

Figure 4 


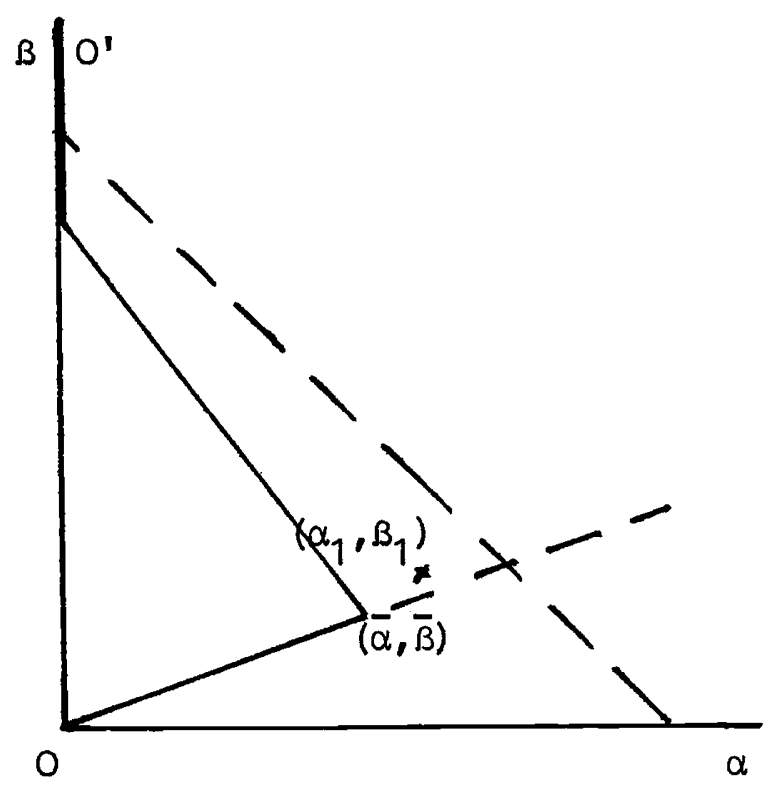

Figure 5

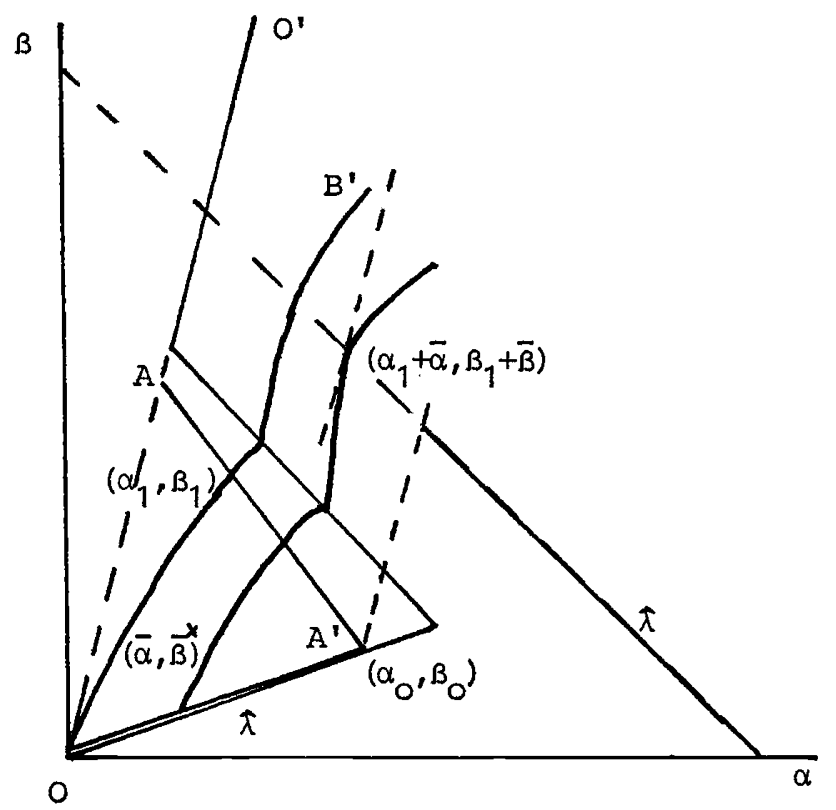

Figure 6 


\section{REFERENCES}

HELPMAN, E., and LAFFONT, J. J. [1975] : "On moral hazard in general equilibrium ", Journal of Economic Theory, 10 (... 1975), 8-23.

HILDENBRAND, W. [1974] : Core and Equilibria of a Large Economy, Princeton University Press, Princeton (New Jersey).

JAYNES, G. D. [1978] : "Equilibria in monopolistically competitive insurance markets", Journal of Economic Theory, 19 (... 1978), 394-422.

KREPS, D. M., and WILSON, R. [1982] : “Sequential equilibria ”, Econometrica, 50 (... 1982), 863-894.

PAULY, M. [1974] : "Overinsurance and the public provision of insurance", Quarterly Journal of Economics, 88 (February 1974), 44-62.

ROTHSCHILD, M., and STIGLITZ, J.E. [1976] : "Equilibrium in competitive insurance markets ", Quarterly Journal of Economics, 90 (November 1976), 629-649.

SHAVELL, S. [1979] : "On moral hazard and insurance ", Quarterly Journal of Economics, 93 (November 1979), 541-562.

WILSON, C. [1977] : " A model of insurance markets with asymmetric information", Journal of Economic Theory, 16 (December 1977), 167-207. 